\title{
Erich Kretschmann as a Proto-Logical-Empiricist: Adventures and Misadventures of the Point-Coincidence Argument
}

\author{
Marco Giovanelli
}

\begin{abstract}
The present paper attempts to show that a 1915 article by Erich Kretschmann must be credited not only for being the source of Einstein's point-coincidence remark, but also for having anticipated the main lines of the logical-empiricist interpretation of general relativity. Whereas Kretschmann was inspired by the work of Mach and Poincaré, Einstein inserted Kretschmann's point-coincidence parlance into the context of Ricci and Levi-Civita's absolute differential calculus. Kretschmann himself realized this and turned the point-coincidence argument against Einstein in his second and more famous 1918 paper. While Einstein had taken nothing from Kretschmann but the expression "point-coincidences", the logical empiricists, however, instinctively dragged along with it the entire apparatus of Kretschmann's conventionalism. Disappointingly, in their interpretation of general relativity, the logical empiricists unwittingly replicated some epistemological remarks Kretschmann had written before General Relativity even existed.
\end{abstract}

Keywords: Erich Kretschmann, Point Coincidence Argument, Moritz Schlick, General Relativity, Logical Empiricism, Conventionalism

\section{Introduction}

In the early 1980s, John Stachel (Stachel, 1980) and John Norton (Norton, 1984) famously shed new light on Einstein's celebrated, yet somewhat cryptic, claim that all physical measurements amount to a determination of space-time coincidences, such as the matching of a pointer with a scale, or, if the world consisted of nothing but particles in motion, the meetings of their world-lines.

In Einstein's published writings, this remark - which Stachel has successfully labeled the "point-coincidence argument" - amounts to the requirement of "general covariance": since all coordinate systems necessarily agree on coincidences, that is, in everything observable, there is no reason to privilege one coordinate system over another. However, Stachel and Norton's archival work on Einstein's private correspondence unmistakably showed that he had introduced the argument in order to identify the fallacy that lurked in his now overwhelmingly famous "hole argument": solutions of the field equations that differ

Email address: marco.giovanelli@uni-tuebingen.de (Marco Giovanelli) 
only by a coordinate transformation agree on point-coincidences - that is, on everything observable - and thus they represent the same physical situation.

This "discovery" has revolutionized the historiographical work on the genesis of general relativity (cf. the volumes of the series Einstein Studies edited by Howard and Stachel and the monumental Renn, 2007, for a recent overall presentation) and engendered a vast and animated debate about its philosophical interpretation that, to this day, has shown no signs of exhaustion (cf. for instance Rickles, 2008, ch. 5, for an effective overview). Another unintended, but no less important consequence of this extensive historical work was to drastically change our perception of early Logical Empiricism. Einstein's "public" point-coincidence remark in his 1916 review paper had been previously regarded as "the beginnings of the empiricist and verificationist interpretation of science characteristic of later positivism" (Friedman, 1983, but see Friedman, 1999, p. 39 ; note 22). During the 1990s, a time of renewed interest in the emergence of logical-empiricist interpretation of general relativity (Howard, 1994; Giere and Richardson, 1996; Friedman, 1999), historical scholarship had an easy time showing that Einstein's point-coincidence argument, considered in the correct setting, could hardly nurture logical empiricists' eagerness "to find heroic precursors" (Howard, 1996, 121) of their verification theory of meaning.

Thomas Ryckman (Ryckman, 1992) pointed out that the logical empiricists' "more geometrico interpretation of the point-coincidence remark" (Ryckman, 1992, 477) was "no more congenial ... than a left shoe to a right foot" (Ryckman, 1992, 496). The truly philosophically revolutionary achievement of general relativity cannot be located in the fact-convention opposition - which was after all a sort of variation of the old Kantian form-content opposition - but precisely in the opposing idea that space and time have no reality independent of the matter or fields within them (Ryckman, 1992). Don Howard (Howard, 1999) suggested that the logical empiricists had failed to "distinguish in principle unobservable, infinitesimal point coincidences from observable, finite pointer coincidences" (Howard, 1999, 493); Einstein considered "coincidences" as physically real "by virtue of their invariance properties", not because they are directly observable (Howard, 1999, 494). Thus also the classical observable-theoretical dichotomy - another watermark of Logical Empiricism - cannot be considered a consequence of Einstein's argument.

The present paper intends to make a contribution to the history of the logical-empiricist misinterpretation of the point-coincidence argument, starting with a historical episode that, as far I can see, has been neglected among historians of the philosophy of science. In the early 1990s Howard and Norton, in a seminal paper (Howard and Norton, 1993) dedicated mainly to the correspondence between Einstein and Paul Hertz (cf. below on p. 37), suggested in passing that an article written by a then-unknown school teacher Erich Kretschmann might have been the unacknowledged source of Einstein's point-coincidence remark (cf. also Janssen, 2007).

Erich Justus Kretschmann (born in Berlin in 1887) had just gotten his doctorate under the guidance of Max Planck by attempting to provide a Lorentzcovariant theory of gravitation (Kretschmann, 1914; see below on p. 6). In 
December 1915 he published a two-part paper with a certain epistemological flavor (Kretschmann, 1915), in which, by relying on the work of Henri Poincaré and Ernst Mach, he argued that only "topological" relations encoded in pointcoincidences are directly accessible to experience (Sect. 3). It was only shortly after the paper was distributed that Einstein started to use the expression "point-coincidences" in private correspondence with Paul Ehrenfest, Michele Besso and Hendrik Lorentz, in order to convince them that solutions of the field-equations that differ only by a coordinate transformation are physically equivalent (Sect. 2). Einstein then abruptly inserted the argument into the quite different mathematical tradition that had culminated in Ricci and LeviCivita's absolute differential calculus (Sect. 4). Kretschmann himself swiftly realized this, and in August 1917 he turned the public version of the pointcoincidence argument against Einstein in a paper that would make him famous (Kretschmann, 1918; Sect. 6).

Considered from this perspective, there is indeed a seldom noticed "ironical attachment" to the logical-empiricist reception of general relativity: in the attempt to unravel the philosophical implications of Einstein's point-coincidence remarks, the founding fathers of Logical Empiricism unwittingly ended up repeating the philosophical reflections entailed in a minor paper that an obscure Gymnasiallehrer had written before general relativity even appeared (Sect. 5). Kretschmann must be credited not only for having possibly suggested to Einstein the expression "point coincidences"; he also anticipated with astonishing clairvoyance the main lines of the philosophy of space and time that Moritz Schlick, Hans Reichenbach and Rudolph Carnap managed to the transform in the 1920s into the only credible philosophical approach to Einstein's new theory of gravitation (Ryckman, 2005, ch. 3). The fact/convention and observable/theoretical distinction and their entanglement with the topological/metrical opposition have a much more humble lineage than the logical empiricists were inclined to believe (Sect. 8).

As we will try to show, Kretschmann's "Proto-Logical-Empircism" provides a clue as to why, in the 1920 s, the logical empiricists' reading of the pointcoincidence argument was so irresistibly convincing, while now it seems irremediably implausible. At the time, philosophically-informed readers could not resist the sort of "Pavlovian reaction" of considering the language of "coincidences" as the heir of the tradition from which Kretschmann had taken it, namely, from the familiar work of authors such as Helmholtz, Poincaré and Mach, etc. The context of the work of Riemann, Christoffel and Ricci in which Einstein had inserted Kretschmann's parlance was far too detached from the main issues of the philosophical debate at the time to be taken into consideration.

Despite conventional wisdom, the claim that only point-coincidences are physically significant could only be properly understood by reading it out of context. When Kretschmann, inspired by Mach and Poincaré, resorted to this turn of phrase he was pointing out the "scarcity" of mathematical structure to which experience and observation have access. On the contrary, Einstein, by appropriating Kretschmann's wording, was dealing with an uncomfortable "abundance" of mathematically different solutions to the field equations that 
was allowed by Ricci and Levi-Civita's mathematical technique (Sect. 4).

To the logical empiricists, by unconsciously reading Einstein's point-coincidence argument as Kretschmann had originally presented it, general relativity indeed appeared to deprive physical reality of all but "topological" properties of the coordinate system. Yet, if any philosophical implication can be attributed to Einstein's theory, it is precisely it is precisely the denial that the bare coordinate system, with its mere "topological" properties, has any independent physical reality. It has only been since the mid-fifties that Peter Bergmann - Einstein's assistant at Princeton since 1936 - restored the spirit of Einstein's notion of "coincidence", by redefining the notion of what is "conventional" and what is truly "observable" in general relativity in a way the philosophical debate has only much later come to appreciate (Sect. 9).

\section{Einstein before Kretschmann: Einstein's Famous Argument against General Covariance}

As is well known, in as early as 1913, after having become familiar with Ricci and Levi-Civita's 'absolute differential calculus' (Levi-Civita and RicciCurbastro, 1900; cf. Reich, 1994), Einstein and Marcel Grossmann were able to outline a theory of gravitation based on the expression $d s^{2}=\sum g_{\mu \nu} d x_{\mu} d x_{\nu}$; the coefficients $g_{\mu \nu}$ represented the numbers by which one has to multiply coordinate differences in order to get real distances as well as the potentials of the gravitational field at a certain point (Einstein and Grossmann, 1913). Einstein and Grossmann were clear from the beginning that the differential equations for determining the quantities $g_{\mu \nu}$, given the distribution of matter and energy - a generally covariant analogon of Poisson's second-order partial differential equation of the gravitational potential - "would likely have the form $\kappa \cdot \Theta_{\mu \nu}=\Gamma_{\mu \nu}$ where $\kappa$ is a constant" (Einstein and Grossmann, 1913, 11). ${ }^{1}$

As is well known, Grossmann had easily found that the four-index object $(i k, l m)$ (known as the Riemann-Christoffel tensor) is the only tensor that contains only the first and second derivatives of the fundamental tensor (cf. Zurich Notebook, p. 14L; CPAE 4, Doc. 10). However, the difficulties in finding a suitable two-index contraction of the Riemann tensor that could be equated to the matter tensor $\Theta_{\mu \nu}$ (Maltese, 1991; Norton, 2007) forced Einstein and Grossmann to publish field equations that were only covariant with respect to linear transformations: an embarrassing result for a theory that intended to extend the relativity principle to all motions by including non-linear transformations of the coordinates. As Einstein admitted on 13 August 1913 in a letter to Lorentz: "the theory refutes its own starting point and is left hanging in the air" (CPAE 5, Doc. 467).

Einstein seems to have become interested at this point in showing that the generally covariant field equations he was unable to find were actually impos-

\footnotetext{
${ }^{1}$ For the sake of historical accuracy, in the course of the paper I will give all formulas in the original notation.
} 
sible. Einstein's argument against general covariance has become enormously famous over the last three decades and has therefore been discussed many times in historical (Stachel, 1980, §§ 3-4; Norton, 1984, § 5), philosophical (Earman, 1989, ch. 9; Earman and Norton, 1987) and physical literature (Rovelli, 2004, $\S 2.2 .5)$. I will provide a rapid overview here, with the aim of introducing some elements that will turn out to be relevant to understanding why Einstein, in late 1915, suddenly became attracted by Kretschmann's point-coincidence language.

\subsection{The Lack of Eindeutigkeit of Generally Covariant Field Equations}

Einstein might have already developed the seeds of an argument against general covariance by the end of August 1913 in a discussion with Michele Besso (Janssen, 2007). In some notes in a manuscript know as the Besso-Memo (Janssen, 2003), Besso imagines a central mass surrounded by empty space, arguing that, due to the arbitrary choice of the coordinate system, the field equations, together with boundary conditions, do not guarantee "a unique determinability of the $g$ 's" in the empty region. Besso suggested that this might not be a problem, since only observable phenomena, such as the motion of particles, should be determined uniquely. Einstein, however, rejected Besso's move as an escape "of no use"; the new solution $K_{2}$ in the coordinate system 2 - he argues - "is also a solution in 1" (Besso Memo p. 2; quoted and translated in Janssen, 2007, ; see Fig. 2 on p. 789, for a facsimile of this passage; see also Renn and Sauer, 2007,241). With this remark - which will turn out to be essential - Einstein probably wanted to show that the trajectories of particles determined by the transformed solution $K_{2}$ will appear different with respect to the original coordinate system 1.

In a footnote of his Vienna lecture, delivered on September 1913, Einstein already hints that he had just found "a proof that such a generally covariant solution to the problem cannot exist at all" (Einstein, 1913). A similar statement can be found in a letter to Ludwig Hopf on 2 November of the same year (CPAE, Vol. 5, Doc. 480). Some days later Einstein explained more explicitly to his friend Ehrenfest - who in 1912 had succeeded Lorentz as professor of theoretical physics at Leiden (Huijnen and Kox, 2007) - that generally covariant field equations cannot achieve "a unique determination [eindeutige Bestimmung] of the $g_{\mu \nu}$ out of the $T_{\mu \nu}$ "; this "is only possible if special coordinate systems are chosen" (CPAE 5, Doc. 484).

To avoid the issue of the additional boundary conditions, Einstein might have inverted Besso's model by imagining an empty region surrounded by matter - hence the label Lochbetrachtung, "hole argument", by which the argument is usually known. The argument was first published in this form on 30 January 1914 in an addendum to a reprint of the Entwurf in the Zeitschrift fur Mathematik und Physik (Einstein and Grossmann, 1914a). Einstein famously considered a region $L$ (which probably stands for Loch) of the "four-dimensional manifold", where no material process occurs, that is, "where the $\Theta_{\mu \nu}$ vanish". Given a solution $g_{\mu \nu}$ of the field equations within $L$, the general covariance of the equations allows us to introduce a new coordinate-system $x_{\mu}^{\prime}$, that coincides with the original coordinate system $x_{\nu}$ at the boundary of $L$ (Einstein and 
Grossmann, 1914a, 260). In the primed coordinates the transformed field $\gamma_{\mu \nu}$ is a solution to the transformed field equations that, in general, is different from the one in the first coordinate system. Einstein concludes that "more than one system of $\gamma_{\mu \nu}$ pertains to the system $\Theta_{\mu \nu}$ " (Einstein and Grossmann, 1914a, 260, my emphasis). Gravitational field equations do not uniquely determine the metric tensor representing the gravitational field.

Interestingly, Kretschmann also formulates the argument similarly in his dissertation Eine Theorie der Schwerkraft in Rahmen der Ursprünglichen Einsteinen Relativitätstheorie (submitted before January 1914, when the oral examination took place). Kretschmann points out that Einstein's theory — where "in place of the scalar potential ten components of a tensor appear" — had encountered a fundamental difficulty: "Mr. Einstein was not able to find a generally invariant form for the differential equations that determine the gravitational field [Schwerfeld] through state quantities of matters" (Kretschmann, 1914, 15); his equations, Kretschmann noticed, are only invariant against linear transformations. Kretschmann interestingly claimed that any attempt to proceed further "must necessarily fail", because in a generally covariant theory "the components of the matter tensor may identically vanish in a certain part of the four-dimensional world ... but not $g_{\mu \nu}$ of the gravitational potentials" (Kretschmann, 1914, 15; my emphasis).

\subsection{A New Field on an Old Coordinate System}

The lack of Eindeutigkeit (cf. Howard, 1991, for the philosphical background of this term) of the generally covariant field equations in this formulation looks like a trivial mistake (Pais, 1982, 222). Actually, "we expect the components of a tensor to change when the coordinates are changed" (Hoffmann, 1982, 101); it is practically the first thing one learns in studying the absolute differential calculus. The Riemann-Christoffel tensor furnishes a univocal mathematical criterion for establishing when the difference of two set of $g_{\mu \nu}$ has, so to speak, substance, and when it is an artifact of the coordinate system chosen.

A more recent reading of the argument (Stachel, 1980/2002; Norton, 1984) has confirmed that Einstein (and Grossmann) might not be charged with such naivety. The clue here is usually considered to be a remark added in a successive version of the argument, written in January 1914 and published on the Physikalische Zeitschrift in 15 February 1914 (Einstein, 1914b). Einstein argues again that even if $\mathfrak{T}_{\mu \nu}=\mathfrak{T}_{\mu \nu}^{\prime}$ outside the hole, in general, inside the hole one might find that $g_{\mu \nu} \neq g_{\mu \nu}^{\prime}$ (Einstein, 1914b, 178). In footnote 1), however, he points out that "the independent variables $x_{\nu}$ on the left side of the equation must be attributed the same numerical values of $x_{\nu}^{\prime}$ on the left side" (Einstein, 1914b, 178; footnote).

This point - which, as we have seen, Einstein had already made in the Besso memo - still did not come out clearly in a successive version of the argument (Einstein and Grossmann, 1914b, 218). However, it became central in the now famous $\S 12$ of Einstein's systematic presentation of the Entwurf-theory that was presented on 29 October 1914 before the Prussian Academy of Sciences: Die formale Grundlage der allgemeinen Relativitätstheorie (Einstein, 1914a). 
Einstein symbolically defined a system of $g_{\mu \nu}$ as functions of the coordinates as $G(x)$. Using this non-standard notation, Einstein makes it clear that the field equations, by means of a coordinate transformation, appear to attribute different field quantities $G(x)$ and $G^{\prime}(x)$ to the same world-point (position-plusinstant-in-time), identified via the same unprimed coordinate system $x$ inside the hole for the same distribution of matter $T_{\mu \nu}$ outside it.

The principle of general covariance appears, then, to be incompatible with physical causality in the loose sense that the same "cause", the same $T_{\mu \nu}$, seems to produce different "effects", or different values of the $g_{\mu \nu}$ at the same point. In the case of electrostatic, for example, it would of course be unacceptable if the field equation, given the same charge distribution, attributed different values of the potentials to the same point, and thus predicted different trajectories for charged particles. In $\S 13$ of his October 1914 Sitzungsbericht, Einstein insisted therefore that - in order to ensure a unique relation between $g_{\mu \nu}$ and $T_{\mu \nu}$ - the class of admissible coordinate systems must be restricted "to justified" transformation between so-called "adapted coordinates" (Einstein, 1914a, 1066ff.; cf. Norton, 1984, §6).

\subsection{The New Meaning of the Eindeutigkeit Requirement}

In January 1915 Lorentz objected to Einstein that by introducing his "adapted coordinates", he had violated the very premise of general relativity, that is, the idea that no preferred coordinate system can be assumed in advance (CPAE 8a, Doc. 43, 70). In his reply, Einstein argued that the "adapted coordinates systems" do not say anything about the geometrical structure of "spacetime", but only about the choice of the coordinate system in an otherwise arbitrarily given space-time (CPAE 8a, Doc. 43, 70). Both a plane and a sphere can be covered with orthogonal coordinates (Cartesian coordinates and latitude and longitude are both orthogonal coordinates). The requirement that the coordinate must be, e.g., orthogonal does not say anything about the geometry of the surface (see also Einstein, 1914c, 348).

Einstein's "Gaussian" parlance shows that he was concerned with the ominous presence of a redundancy allowed by the formalism of absolute differential calculus. Einstein's initial reaction was to restrict our freedom to relabel the space-time coordinates in order to save the theory's predictability. In August 1915, the Göttinger Physicist Paul Hertz; cf. Howard and Norton, 1993), resorting again to Gauss's notation, may have suggested to Einstein that such a redundancy might not have been a problem at all. The apparently different solutions of the field equations considered by Einstein - a point that will turn out to be relevant in the following sections - are simply "developable" onto each

other. They can be deduced from one another by a deformation that preserves all lengths and thus represents the same space-time geometry. Einstein was, however, not really convinced by Hertz's argument: "So once again: I would not think of requiring that the world should be 'developable onto itself,' and I do not understand how you require such a dreadful [so etwas fürchterliches] thing of me" (CPAE 8, Doc. 108, 161; tr. in Howard and Norton, 1993, 46) 
Hertz's suggestion probably reflects a familiarity in the use of arbitrary coordinates, which might have been widespread in the Göttingen community formed around David Hilbert (Howard and Norton, 1993; Brading and Ryckman, 2008), who, at about that time had started to work intensively on Einstein's theory (Einstein had just visited Göttingen between June and July of 1915; Corry 2003). It is hard to establish precisely when Einstein came to realize that two sets of coefficients which are "developable onto another" - that is, that differ only by a coordinate transformation - represent the same physical situation.

However, under the pressure of competing with Hilbert (Mehra, 1974; Earman and Glymour, 1978; Sauer, 1999; Renn and Stachel, 2007; Brading and Ryckman, 2008), by November 1915 Einstein had regained general covariance for the field equations (Pais, 1982; Norton, 1984; Janssen and Renn, 2007; Renn and Sauer, 2007), which he presented in four communications to the Prussian Academy (Einstein, 1915c,d,a,b): "a real triumph of the method of the general differential calculus founded by Gauss, Riemann, Christoffel, Ricci, and Levi-Civiter [sic]" (Einstein, 1915d, 778), as Einstein famously put it.

As the so-called perihelion paper (published on 25 November) most clearly reveals, Einstein was suddenly ready to accept that "the $g_{\mu \nu}$ ", for the given mass distribution, are "not yet mathematically fully determined" through the field equations, which allow an entire class of different solutions that differ only by a coordinate transformation: "One may be justified, however, in assuming that all of these solutions can be reduced to one another by means of such transformations, and that (for the given boundary conditions) they therefore differ from one another only formally, but not physically" (Einstein, 1915a, 832). Different sets of $g_{\mu \nu}$ that differ only by a coordinate transformation are mathematically different, but represents the same physical situation; their difference is only an artifact of the coordinate system chosen. Einstein now considered it fully acceptable "to obtain a solution without considering the question whether or not it is the only unique possibility" (Einstein, 1915a, 832; tr, Howard, 1996, 467; my emphasis).

On 14 December 1915 Einstein could then proudly announce to Schlick who had just sent him his paper on special relativity (Schlick, 1915) — his "newly found result": a theory, whose generally covariant equations, has deprived space and time of the "last vestige of physical objectivity" (CPAE 8, Doc. 165, 221). Einstein, however, might have felt he owed an explanation to his friends and correspondents, such as Ehrenfest, who over the previous two years he had insistently tried to convince that covariant field equations were impossible precisely due to their lack of univocalness or Eindetutigkeit. It was at the end of 1915 that, from an unexpected source, Einstein may have found an apt "rhetorical device" to escape from his own argument against general covariance.

\section{Kretschmann's Early Point-Coincidence Argument}

On 15 October 1915 Kretschmann - at the time a substitute teacher in a Königsberg Gymnasium — had finished writing a rather convoluted paper in two 
parts, which was entitled: Über die prinzipielle Bestimmbarkeit der berechtigten Bezugssysteme beliebiger Relativitätstheorien (Kretschmann, 1915). The Annalen der Physik received Kretschmann's first post-doctoral paper on 21 October 1915. The paper contained, among others things, an argument that unbeknownst to its inventor - would enjoy an enormous popularity in the history of physics and philosophy of science, but also, as we shall see, would give rise to a long series of serious misunderstandings:

Each measurement of spatiotemporal quantities, such as length, time, volume, speed etc., is performed in a way that certain material or immaterial parts (i.e. light rays) of the measuring instrument are completely or only partially brought to spatiotemporal superposition with parts of the object to measure. The result of measurements is obtained by transporting the given spatiotemporal measure-relations between the parts of the measuring instrument and those of the measured object, with which they have been brought to superposition. What is observed here - if we neglect, at first, all direct metrical determinations - is only the complete or partial spatiotemporal coincidence [Zusammenfallen] or non-coincidence [Nichtzusammenfallen] of parts of the measuring instrument with parts of the measured object. Or more generally: topological relations between spatiotemporally extended objects ... In as much as the spatiotemporal relations consist in measure-relations among spatiotemporal quantities, they cannot be traced back to purely observational facts, even by checking the given measuring instruments through other instruments. Exclusively new topological relations are observed again (Kretschmann, 1915, 914).

Kretschmann readily admits that he came to this conclusion by resorting to "E. Mach's and H. Poincaré's analyses of physical experience" (Kretschmann, 1915, 911). According to Kretschmann, Mach and Poincaré have shown "that it [the experience] can deliver only spatiotemporal relations of topological nature [von bestimmten räumlich-zeitlichen Beziehungen nur solche topologischer Art liefern kann]", i.e. precisely the relations of coincidence or non-coincidence of points (Kretschmann, 1915, 911). On the contrary, according to Kretschmann, "all topologically independent measure-determinations [Maßbestimmungen] of spatiotemporal quantities should be regarded as empirically unprovable results of physical theories" (Kretschmann, 1915, 912).

Kretschmann must have had some interest in philosophy. In the brief Lebenslauf, which is included in his dissertation, he claims to have attended (among those of many others) the lectures of Alois Riehl in Berlin (Kretschmann, 1914, backcover), who, in his widely read monograph on Kant, also used the language of coincidences to explain the origin of our space representation (cf. Riehl, 18761887, vol. 2, part I, 145 (1879); see also Riehl, 1908-1926, vol. 2., 175 (1925)) . Kretschmann's sources are highly interesting from a historical-philosophical point of view; it is worth paying some attention to the passages to which Kretschmann explicitly refers us:

- Ernst Mach, Erkenntnis und Irrtum, Leipzig 1906 (actually Mach, 1905); In particular Kretschmann mentions the chapters: "Der physiologische 
Raum im Gegensatz zum metrischen", pp. 337ff., and "Die physiologische Zeit im Gegensatz zur metrischen", pp. 423ff. Here Mach claims that, starting from the information provided by our senses, "one could build at most a topology". The physiological space and the geometrical space are in fact profoundly different; however, they have a few qualities in common: "Both spaces are threefold manifoldnesses. To every point of geometric space $A, B, C, D \ldots$ corresponds a point $A^{\prime}, B^{\prime}, C^{\prime}, D^{\prime} \ldots$ of the physiological space. If $C$ lies between $B$ and $D$, there is also $C^{\prime}$ between $B^{\prime}$ and $D^{\prime \prime \prime}$, (Mach, 1905, 337; tr. Mach, 1906, 11). In particular Kretschmann read Mach in the sense that experience can only provide univocal information about the "contact" or non-contact of bodies - also distant perceptions (such as sight and hearing) can be at least reduced to the sensation of contact - and nothing else.

- H. Poincaré, Wissenschaft und Hypothese, Leipzig 1906 (Poincaré, 1906a, German translation of Poincaré, 1902), part 2, chap. 4 and 5, p. 52ff., Letzte Gedanken, Leipzig 1913 (Poincaré, 1913b German translation of Poincaré, 1913a), chap. 2 and 3, p. 33ff; Der Wert der Wissenschaft, Leipzig 1906 (Poincaré, 1906b German translation of Poincaré, 1905, chap. 2 u. 3, p. 26ff. In particular, Kretschmann seems to be interested in Poincaré's claim that space, when considered independently of our measuring instruments, has only topological properties (that is, those studied in analysis situs), but it is deprived of any metric or even projective structure. "It is amorphous, that is, it does not differ from any space which one can derive from it by any continuous deformation whatever" (Poincaré, 1913b, 60; Fr. orig. Poincaré, 1913a, 62; Eng. tr. Poincaré, 1963, 27), since bodies that are in "contact" before the transformation will still be in contact after the transformation. Poincare translated this intuitive way of thinking in the abstract language of coordinates. The contact that, let's say, the finger feels when it touches an object, means that at a certain time $t$ the coordinates $x, y, z$ are the same for both the finger and for the object: $x=x^{\prime}, y=y^{\prime}, z=z^{\prime}$ (Poincaré, 1913b, 60; Fr. orig. Poincaré, 1913a, 83; Eng. tr. Poincaré, 1963, 83) . The analysis situs or topology considered indistinguishable those spaces that are mapped into each other so that $x^{\prime}, y^{\prime}, z^{\prime}$ are three continuous functions whatever of $x, y$, and $z$. In his more technical papers, published at the turn of the century, Poincaré had made clear that these functions must "have continuous derivatives" (Poincaré, 1895, 198). Poincaré labeled such transformations "homeomorphisms" (Poincaré, 1895, §2 whereas we would rather speak of "diffeomorphisms"; cf. Moore, 2007). The set of such transformations form a group that is "one of the most general which can be imagined", which Poincaré - in a parlance somewhat different from ours - considered the object of the analysis situs or topology.

Kretschmann's strategy is then fairly simple: he extended Mach and Poincaré's insistence on the "topological" determinacy and metrical amorphousness of space to the four-dimensional framework (see of course Minkowski, 1909, but 
also Poincaré, 1906c), that was by that time fully implemented in the physical literature. Kretschmann's premises are the following:

- In order to be free from "every presupposition of a given form a priori of spatial intuition" (Kretschmann, 1915, 916f.), Kretschmann considered a "spatiotemporal physical system of reference [Bezugssystem der Physik] as a - four-dimensional - manifold of pure numbers" (Kretschmann, 1915, 917). Kretschmann is committed to the idea that space-time is the set of all possible quadruples of real numbers; a world-point is a quadruple of real numbers, etc. The neighborhood relations among world-points can be expressed in a rigorous way, without resorting to geometrical intuition: events that coincide are assigned the same quadruple of coordinate numbers and adjacent events correspond to adjacent values of the coordinates (Kretschmann, 1915, §§5, 6, 22). The set-theoretical definition of "neighborhood" (Hilbert, 1902, 234-235; Hilbert, 1903, app. IV, 122-123.) via the notion of "open set" (Weyl, 1913, 11f.) and the separability axiom (Hausdorff, 1914, 214f.) was still considered avant-garde at that time (e.g. Study, 1914 still resorts to the definition of a manifold as a number manifold).

- Kretschmann recognizes the necessity of introducing what he calls "mapping postulates [Abbildungspostulate]" (Kretschmann, 1915, 918) to assure law-like connections between the abstract number-manifold and the empirical world: "If the space-time coordinates are nothing else than a system of pure numbers" then "there is no connection a priori between them and some magnitudes of the empirical space and the empirical time" (Kretschmann, 1915, 917).

Such "mapping prescriptions [Abbildungsvorschriften]" a) must be independent from a space and time location as well as from the particular condition of the observer. (Kretschmann, 1915, 918) b) must reproduce the "same topological properties and relations" (number of dimensions, type of connection etc.) of the phenomenal world (Erscheinungswelt) in "the system and manifold of pure (coordinates)-numbers" (Kretschmann, 1915, 918f.)

In order to achieve this result, Kretschmann assumes that it is legitimate to systematically substitute observable finite point-coincidences with infinitesimal point-coincidences, that is, to assume that "all topological observations are valid with absolute exactness and sharpness" (Kretschmann, 1915, 912; cf. §15). This implies "the elimination of the unavoidable complications that arise from the interpretation of macroscopic observations in the light of an atomic theory of matter" (Kretschmann, 1915, 912; cf. $\S 80)$.

Given these premises, Kretschmann argues that "the most general and the most fundamental law for the mapping of the world on the reference system" is the following: "starting from topological observations" one can only establish that 
"all points of the phenomenal world constitute a simply connected manifold ${ }^{2}$ of exactly four spatiotemporal dimensions" (Kretschmann, 1915, 924).

Kretschmann must have sensed that he was somehow begging the question; one can reach the conclusion that such a weak topological structure is accessible to experience only by resorting to "principles that are not empirically provable [empirisch doch nicht erweisbarer Sätze]", that is "the mapping postulates" (Kretschmann, 1915, 911); so it is not clear if experience "discovers" the topological structure of the world, or, as a Kantian might have objected, if this structure has been put into experience via the mapping postulates.

However, Kretschmann, after having recognized the impossibility of eliminating every non-empirical element from his analysis (Kretschmann, 1915, 919f.), could congratulate himself for having showed that observation provides at most topological information about the phenomenal world - that is, information about the coincidence or non-coincidence of points - and nothing more. Everything else - in particular "Ispatial-temporal measure relations" (Kretschmann, 1915, 924) - are "inaccessible to observation" and "go beyond what can be controlled through observation" (Kretschmann, 1915, 924).

Consequently, two mappings of the world that can be smoothly deformed onto one another by distorting the lengths of lines and the angles between them, while preserving point-coincidences, are empirically indistinguishable:

The line of thought [Gedankengang] just presented can be briefly summarized as follows: Each statement about spatiotemporal measure-relations - apparently gained by mere observation - can be interpreted as the expression of purely qualitative topological observational facts, which have been arranged in a simple form by the (unconscious) addition of quantitative theoretical assumptions. If one accepts this interpretation, it follows that in no case, through mere observation, can a soundly-based decision be made between two qualitatively different but topologically identical mappings of the world of appearance onto a space-time reference system. (Kretschmann, 1915, 916).

The topological relations of space-time to which experience has access are not rich enough and do not furnish any sufficient reason to establish which one of the topologically equivalent but metrically different mappings of the world is the "true" one: statements that "cannot be reduced to purely topological relations can be regarded as mere - or at most methodologically justified [hochtens methodologisch begründete] - conventions. However, one cannot say that all spatiotemporal measure-relations ... are conventions. Rather, this claim only applies to the topologically independent ones among them" (Kretschmann, 1915, 924; my emphasis).

This result is most apparent if one considers that every space-time theory, according to Kretschmann, is characterized by a set of coordinate transformations that let some "algebraic expression" (cf. Kretschmann, 1915, §§18-21) between coordinates be unchanged when one substitutes the unprimed coordinates with

\footnotetext{
2 Roughly a manifold without holes
} 
the primed ones. The set of these transformations forms a group - for each transformation there exists an inverse contained in the set, and the composition of any two transformations of the set in turn belongs to the set (Kretschmann, 1915, 925; footnote 1). The group of transformations in which the laws of physics are invariant is decided by the relativity principle of the theory (probably the most significant of Kretschmann's results). A choice among different possible, physically different relativity theories - that is, theories with laws that are invariant against some sub-group of the general group of smooth coordinate transformations - remains under-determined by the topological structure accessible to experience.

Kretschmann analyzed in detail theories that were characterized by a finiteparameter continuous group of transformations: the absolute theory (which admits an absolute speed respect to the aether, such as Abraham's theory; cf. Kretschmann, 1915, §§51-53), the Galilei-Hertz Relativity Theory (which incorporates Heinrich Hertz's aether-dragging hypothesis; cf. Kretschmann, 1915, $\S 53)$ and that of the Lorentz-Einstein Relativity Theory (with time-dilatation and length-contraction; cf. Kretschmann, 1915, §§58-61). Additionally, Kretschmann takes into consideration theories based on infinite-parameter groups of transformations, such as the Mach-Reissner Theory (a Machian explanation of inertia; cf. Kretschmann, 1915, §63) and the "new Einstein theory" (that is, the Entwurf-theory; cf. Kretschmann, 1915, §64), which Kretschmann addresses only very briefly at the very end of the paper. No mention is made of Einstein's argument against general covariance, which, as we have seen, Kretschmann knew about (see above on p. 6).

Kretschmann's main concern was in fact totally different: all the above-listed theories agree on the "purely topological content verifiable through observation [durch Beobachtungen verifizierbaren]" (Kretschmann, 1915, 938), that is, they agree on point point-coincidences. All "topologically independent" measurerelations "can only be made true by convention" (Kretschmann, 1915, 939). In particular, a stipulation about the behavior of what Kretschmann calls "ideal instruments" (paths of light rays and freely moving bodies, and rigid bodies and clocks) is needed.

Kretschmann concludes that "through mere experience (observation and induction) essentially only topological relations for the determination of the system of reference can be provided" (Kretschmann, 1915, 979), and even then, "only with the help of certain purely theoretical propositions, the mapping postulates, that put into relation the empirical space and the empirical time with the spaceand time-coordinates of theoretical physics" (Kretschmann, 1915, 979). Consequently, "it is not possible to pick out a single [class] of systems of reference from among those that are compatible with the mapping postulate, that is, from those that can be mapped onto one another through arbitrary continuous transformations, without resorting to 'conventional' theoretical measure-determinations and arbitrary stipulations which go beyond experience" (Kretschmann, 1915, 979).

Kretschmann claims that, of all the possible topologically equivalent sets of measure-relations, one can be privileged only by resorting to "thought-economical 
principles" [denkökonomischen Sätze] (Kretschmann, 1915, 982, an undeniably Machian wording; cf. e.g. Banks, 2003, ch. 8: "If at all," he argues, "the (empirically provable) topological laws of a phenomenon always have to be equipped [einzukleiden] with measure-relations in the simplest way possible" (Kretschmann, 1915, 982). Kretschmann's epistemology starts to sound very familiar: In physical theories we can distinguish a factual element verifiable by experience on which a class of empirically equivalent theories agree (pointcoincidences), and a conventional element (metric-relations) determined pragmatically as leading to the "simplest" theory in the class. Of course, Kretschmann is aware that it is hard to say "how this simplest outfitting [Einkleiden] of topological laws in metrical relations is in general defined" (Kretschmann, 1915, 982, n. 3). However, it is at least undeniable that "simplicity" has nothing to do with "truth".

\section{Einstein After Kretschmann: The "Private" and the "Public" Point-Coincidence Argument}

\subsection{The "Private" Point-Coincidence Argument}

Kretschmann's paper appeared in the Annalen der Physik on 21 December 1915; on 26 December Einstein wrote a letter to Ehrenfest which has been referred to in the literature many times in the last thirty years. Einstein briefly reminded Ehrefenst of the problem raised in the above-mentioned $\S 12$ of his 1914 Sitzungsbericht - the possibility of referring two solutions $G(x)$ and $G^{\prime}(x)$ to the same coordinate system $x$. "In place of $\S 12$," he continues, one must introduce the remark that "[t]he physically real in the world of events [Weltgeschehen] ... consists in spatiotemporal coincidences [Koinzindenzen]" - such as "the intersections of two different world-lines" — "and nothing else!" (as he added in a footnote). In particular, "two systems of the $g_{\mu \nu}$ " which can be obtained from one another "through mere space-time transformation" must be regarded as "completely equivalent [gleichbedeutend]"; in fact, "they have all spatiotemporal point-coincidences in common, i.e., everything that is observable", "in contrast to that which is dependent upon the choice of a reference system" (CPAE 8, Doc. 173,228$)$

Apart from the use of the Latin-rooted word Koizindenz in place of its Germanic equivalent Zusammenfallen, ${ }^{3}$ Einstein's wording clearly resembles that of Kretschmann's. It is "extremely suggestive" (Howard and Norton, 1993, 53) that the argument made its sudden appearance in this letter to Ehrenfest dated only five days after the distribution of Kretschmann's paper; there is no trace of it in Einstein's public and unpublished work before this date (Howard

\footnotetext{
${ }^{3}$ The German word reproduces the etymological structure of the Latin word: cum = mit; incidere = fallen: "fall together". According to Howard and Norton 1993, however, Koinzidenz is more suggestive of the "notion of the intersections of lines at extensionless points, whereas Zusammenfallen is "more suggestive of macroscopic congruences" of measuring instruments (Howard and Norton, 1993, 54)
} 
and Norton, 1993, 53). It is plausible to conjecture, then, that Einstein deftly modified Kretschmann's argument without acknowledging his source in order to justify to his correspondents that his initial concerns about general covariance were unfounded.

Einstein has often been accused of reluctance in recognizing the influence of other scientists on his work. ${ }^{4}$ However, in this case, it must be strongly emphasized that Kretschmann's argument in Einstein's hands became something totally different from its original version. Kretschmann, following Mach and Poincaré, had been considering space-times that are smoothly deformable onto each other and thus endowed with different sets of measure-relations. Lines of the same length may not have equal length after the transformation; only the "topological" properties, represented by their points of intersection, remain unchanged.

Einstein, in contrast, inserted Kretschmann's argument into the context of Ricci and Levi-Civita's absolute differential calculus. He considered two $g_{\mu \nu^{-}}$ systems that differ only by a coordinate transformation, that is, the very same spatiotemporal measure-relations in different coordinate systems: lines of equal length still have equal length, angles between them at points of intersection will remain unchanged, and thus a fortiori the points of intersection of such lines will also remain unaltered.

This mathematically triviality might however become problematic if one regards the $g_{\mu \nu}$ as potentials of a physical field. In traditional field theories, one usually first covers the space with a coordinate grid and then distributes the field potentials on it, from which the possible trajectories of charged particles can be derived. In general relativity, however, a new set of potentials $g_{\mu \nu}$ may be introduced by virtue of a simply coordinate transformation. The world-lines of light rays and massive particles that passed through certain points of the original coordinate grid will then follow different paths in the transformed field, i.e. will intersect different grid points.

Put this way, it then becomes understandable why Kretschmann's pointcoincidence remark might have suddenly appeared to Einstein as a "godsend" (Janssen, 2007, 827). Using Kretschmann's turn of phrase, one can effectively put a finger on the conceptual error that lurks in this seemingly plausible reasoning. As we have seen, in 1913 Besso had already suggested that only observable phenomena, such as the paths of particles, should be considered relevant. It might not be mere chance that Einstein wrote to Besso on 3 January 1916 that it was not the movements of material points that are observable, but only "the points of intersection of their world lines". The laws of nature do "not determine more than the totality of the spatiotemporal coincidences" (CPAE 8, Doc. 178, 235).

It is worth lingering on Einstein's word choice in these famous passages in order to understand the profound difference between Kretschmann and Einstein's

\footnotetext{
4 "The secret to creativity is knowing how to hide your sources", is often quoted but probably apocryph statement included in many collections of Einstein quotations.
} 
versions of the point-coincidence argument. Of course, it is simply not true that only point-coincidences are physically relevant in general relativity, and nothing more than point-coincidences. Two sets of $g_{\mu \nu}$ that differ only by a coordinate transformation obviously agree not only in such coincidences, but also in the length of world-lines and angles between them at the point of intersection, in the distinction between geodesics and non-geodesics, etc. As Hertz had already pointed out to Einstein in August 1915, the two solutions are "developable" onto one another.

Einstein therefore had come to appreciate Kretschmann's turn of phrase, for reasons that have nothing to do with Kretschmann's metrical conventionalism. When Einstein maintains that only the intersections of world-lines have a physical content, he is emphasizing that the question of where they intersect is not well-posed. It is physically meaningless to argue that the path of two worldlines determined by inter-transformable $g_{\mu \nu}$-sets do not meet at the same point identified by their coordinates; the same point is precisely where the two worldlines meet, information that is "naturally preserved under all transformations" (CPAE 8, Doc. 178, 235).

Using Kretschmann's wording, Einstein could then show his correspondents pictorially that in general relativity points cannot be identified by their coordinates independently from the field. In traditional field theories, if one removes the field potentials the bare coordinate system remains; in general relativity, by removing the $g_{\mu \nu}$, nothing is left. The field quantities, that is, the $g_{\mu \nu}$, not only define the physical field and determine the paths of light rays and massive particles; they also encode all information about the coordinate system in which they are presented. The gravitational potentials and the coordinates system are inextricably entangled in general relativity and one cannot describe one without the other (cf. Norton, 2005, §5.5.3).

The strategy of referring a primed solution of the field equations to the unprimed coordinate system was probably felt as instinctively correct. As has been shown (Kox, 1987), Lorentz was also clearly affected by the same prejudice. In a letter to Ehrenfest on 9 January 1916, Lorentz resorted to similar reasoning: in the case of a 'matter-free' field, he pointed out, if one introduces by virtue of a coordinate transformation a new solution of the field equations (A) "symbolically expressed" as $g_{\mu \nu}^{\prime}=F^{\prime}\left(x_{\alpha}^{\prime}\right)$, [t] hen $g_{\mu \nu}=F^{\prime}\left(x_{\alpha}\right)$ will also satisfy equations $(A)$ ". (Lorentz to Ehrenfest, 9 Jan. 1916 (cited and translated in Kox, 1987).

On 10 January 1916, the day after having sent this remark to Ehrenfest, Lorentz received a letter from him. Attached he found Einstein's 9 January 1916 epistolary response to objections Ehrenfest had made in a (lost) letter of 5 January 1916. Einstein famously resorted to a diagram representing the geodesic path of a light ray coming from a distant star that passes through an aperture directing it at a right angle onto a photographic plate. Then he suggested to Ehrenfest that he copy the diagram onto tracing paper, bend it without stretching it, and then make a carbon copy back onto the writing paper.

"When you relate the figure once again to orthogonal writing paper coordinates", Einstein explained to Ehrenfest, the transformed situation is "math- 
ematically a different one from before, of course also in relations to the $g_{\mu \nu}$ " (CPAE 8, Doc. 180, p. 238; my emphasis); however "physically it is exactly the same since the writing paper coordinate system is only something imaginary [eingebildetes]" (CPAE 8, Doc. 180, 239; my emphasis); it has no reality independent from the $g_{\mu \nu}$. It is physically irrelevant at which point on the writing paper the world-line of the light ray intersects the world-line of the pinhole; the point being referred to is, in all coordinate system, where the light ray intersects the pin-hole: "It all depends on coincidences" (CPAE 8, Doc. 180, 239), Einstein restated to Ehrenfest; the coordinates of such coincidences have no physical meaning.

Einstein's "Kretschmannian" coincidence parlance achieved the hoped-for result. Lorentz read Einstein's letter to Ehrenfest and was immediately convinced: "I had read only a part of it", he wrote on 10/11 January 1916, "and I saw that he was entirely right. I wrote to him straight away to retract my objections of yesterday" (cited and translated in Kox, 1987, 73). Einstein wrote to Ehrenfest on 17 January expressing his joy for the extraordinary receptivity of the Leyden community of physicists: "a little corner of heaven on this dreary planet" [Prachtswinkel auf diesem öden Planeten], as he famously described it. On the same day Einstein could write to Lorentz: "I am ... very happy that you agree with me; in particular I see that you have thought over the theory entirely and have familiarized yourself with the idea that all of our experiences in physics refer to coincidences" (CPAE 8, Doc. 245). In the same letter Einstein completely agrees with Lorentz's formulation of the general covariance and suggested that Lorentz make his considerations available to "other physicists, too, by writing a treatise on the foundations of the theory" (CPAE 8, Doc. 183, 245; cf. Illy 1989).

\subsection{The "Public" Point-Coincidence Argument}

As early as 26 February 1916 - at the meeting of the Dutch Academy of Science - Lorentz presented a coordinate-free treatment of general relativity, which he described precisely in terms of point-coincidences. He explicitly referred to a "striking remark" (Lorentz, 1917, 1342) of Einstein — "[in] a correspondence I had with him" (Lorentz, 1917, 1342, footnote 3) - "that the coincidences only are of importance" (Lorentz, 1917, 1343), whereas the "coordinates will be of secondary importance" (Lorentz, 1917, 1344). Lorentz appears to have been the very first to refer publicly to the point-coincidence argument (cf. Illy, 1989; Janssen, 1992, for more details).

The most famous "public version" of the argument appeared of course in Einstein's review article on general relativity received by the Annalen der Physk on March 1916 (Einstein, 1916). Einstein, after having motivated the need to generalize the restricted principle of relativity, famously suggests, as an independent argument for general covariance, that all physical experience can be

reduced to "nothing but verifications of such meetings of the material points of our measuring instruments with other material points". Since "all our physical experience can be ultimately reduced to such coincidences", then "there is 
no immediate reason for preferring certain systems of co-ordinates to others", because all coordinate systems agree in point-coincidences (Einstein, 1916, 776).

Without any further details, Einstein's argument dangerously resembles Kretschmann's original version. Einstein seems to defend an impoverished image of physical reality where only point coincidences are physically relevant, since only point-coincidences are preserved by the general group of smooth coordinate transformations; everything else is apparently deemed to be physically insignificant. Space and time are deprived of "the last remnant of physical objectivity", as Einstein famously put it (Einstein, 1916). This reading of the argument although perhaps in consonance with Einstein's "subtractive strategy" (Norton, 1999) — is undoubtedly misleading.

To find a less ambiguous "public" formulation of the point-coincidence argument, one has turn again to the Leyden community of physicists, where the issue must have provoked a lively discussion. In July 1916, in his detailed review of Einstein's general theory (the first one that appeared in English in October 1916), Willem De Sitter, — who would become famous for his fundamental contributions to general relativistic cosmology - formulated the point-coincidence argument in a way that makes the issues at stake much more clear:

They [the field equations $G_{i k}=\kappa T_{i k}$ ], therefore, are not sufficient to determine the $g_{i k}$. This is essential. For the $g_{i j}$ determine the character of the four-dimensional system of reference, and the principle of general relativity requires that this system can be arbitrarily chosen. They must, therefore, not imply a choice of the system of co-ordinates, i.e. they must leave the $g_{i j}$ to a certain extent undetermined. In order to determine the $g_{i j}$ completely we must add to the equations $\left[G_{i k}=\kappa T_{i k}\right]$ four additional conditions, which can be arbitrarily chosen, and which determine the choice of the system of reference. According to the form of these additional conditions the $g_{i k}$ will be different functions of the co-ordinates, the equations of motion will be different, the course of rays of light will be different, but there will always be the same intersections of world-lines, and consequently all observable phenomena will be exactly the same: they will only be described by a different system of space co-ordinates and a different time. One system may be more convenient than another — this is a matter of taste; but we cannot say that one system is true and another false (de Sitter, 1916b, 418f.; my emphasis).

De Sitter's formulation leaves no room for misunderstanding. He makes clear the obvious fact that, in general relativity, the smooth "deformation" of the coordinate system must be accompanied by the change of the $g_{i k}$, so that the two situations agree not only in point-coincidences, but also in the lengths of world-lines and the angles of intersections among them. The claim that "what we observe are always intersections of world-lines", should be read in the sense that we observe only that the world-lines meet, not where they meet: "About the course of world-lines between the points of intersection we know nothing, and no observation can ever tell us anything" (de Sitter, 1916a, 700).

Referring to Hilbert's "first communication" on the "foundation of physics" (Hilbert, 1915), in August 1916 De Sitter emphasized that "an essential fea- 
ture of the new theory is that of the ten equations $\left(G_{i k}=\kappa T_{i k}\right)$, only six are mutually independent. The $g_{\mu \nu}$ are therefore not determined by these equations: there remains a large amount of freedom unless four additional equations are imposed. It was precisely the "large amount of freedom" (de Sitter, 1916a, 708)** mentioned by De Sitter that Einstein initially thought conflicted with the theory's predictability. The problem arises because in general relativity the physical system being dealt with is described by more variables than there are physically independent degrees of freedom.

In his "second communication" (presented on December 1915 and February 1916 published as Hilbert, 1917), Hilbert famously presented this issue in terms of the Cauchy problem for the field equations: given the initial data, Einstein's equations leave undetermined the future evolution of four out of the ten values of the $g_{\mu \nu}$ (Stachel, 1988/1992). Hilbert showed that this is not in conflict with the law of causality by resorting — as his Göttingen colleague Paul Hertz did in August 1915 - to the language of "Gaussian coordinates" (on Hilbert and Hertz see Corry, 2003, 326).

Just like in the "theory of curves and surfaces", "so also in physics we must characterize a statement that does not remain invariant under any arbitrary transformation of the coordinate system as physically meaningless" (Hilbert, 1917, 61). For instance - as Hilbert pointed out in his November 1916 "causality lecture" - in geography the assertion "that three places have the same geographical latitude is meaningless; in fact this claim is destroyed by a coordinate transformation" (Hilbert, 1916/1917, 8). On the contrary, the claim that "three places lie on a great circle of the earth is independent of the choice of reference system" (Hilbert, 1916/1917, 8; on the relation to the "hole argument", see Renn and Stachel, 2007; for a different interpretation see Brading and Ryckman, 2008, §7). Intersecting great circles intersect in every projection of the earth on a flat sheet of paper; the fact that, in different projections, they appear to intersect at different points of the paper is, needless to say, irrelevant to the cartographer. The point being referred to can be identified in all projections, as can the point where the two great circles meet: "any individual assertion regarding an occurrence [Begebenheit] or an encounter [Zusammentreffen] of occurrences has a meaning, if is independent of designation [Benennung], if it is invariant" (Hilbert, 1916/1917, 5).

\section{Between Kretschmann and Einstein: Schlick's Point-Coincidence Argument}

Thus, Einstein's formulation of the point-coincidence argument in his 1916 review article was utterly elliptical. The ambiguity resides precisely in the claim that only point-coincidences have physical reality, that is only the fact that two or more world-lines have "a particular system of co-ordinate values, $x_{1}, x_{2}, x_{3}, x_{4}$ in common" (Einstein, 1917, 64). As we have tried to show, Einstein borrowed Kretschmann's expression in order to explain to his correspondents that the particular coordinate values of such common points are not relevant to physics. However, it is understandable that Einstein's statement could 
be instinctively read as Kretschmann had originally formulated it in 1915. If only point-coincidences are relevant, then all remaining properties of spacetime that are not reducible to point-coincidences cannot find expression in the fundamental mathematical structure of the theory and thus are arbitrary and conventional.

\subsection{Schlick's 1917 Paper on General Relativity}

This was precisely the conclusion reached by Moritz Schlick in his celebrated Raum und Zeit in der gegenwärtigen Physik, published in two installments on 17 March and 23 March 1917 in the semi-popular journal Die Naturwissenschaften (Schlick, 1917a, tr. in Schlick, 1978, vol. I, 207-269). As is well known, Schlick was trained as a physicist, and on 20 May 1904 - exactly ten years before Kretschmann - he had also completed his doctorate in physics under the direction of Max Planck (Schlick, 1904).

Schlick claims to follow Poincaré's lead by declaring that, if "the objects in the universe are arbitrarily distorted in arbitrary directions", as long as we suppose that "all measuring instruments, including our own bodies" share the same deformation, "the whole transformation immediately becomes unascertainable" (Schlick, 1917a, 164, tr. 1978, I, 227). Schlick justifies this claim by resorting to the idea that only coincidences are ascertainable by those measuring instruments:

The adjustment and reading of all measuring instruments ... is always accomplished by observing the space-time-coincidence of two or more points ... Such coincidences are therefore, strictly speaking, alone capable of being observed; and the whole of physics may be regarded as a quintessence of laws, according to which the occurrence of these spacetime-coincidences takes place. Everything else in our world-picture which can not be reduced to such coincidences is devoid of physical objectivity, and may just as well be replaced by something else. All world pictures which lead to the same laws for these point-coincidences are, from the point of view of physics, in every way equivalent. We saw earlier that it signifies no observable, physically real, change at all, if we imagine the whole world deformed in any arbitrary manner, provided that after the deformation the co-ordinates of every physical point are continuous, single-valued, but otherwise quite arbitrary, functions of its co-ordinates before the deformation. Now, such a point-transformation actually leaves all spatial coincidences totally unaffected; they are not changed by the distortion, however much all distances and positions may be altered by them. For, if two points $A$ and $B$, which coincide before the deformation (i.e. are infinitely near one another), are at a point the coordinates of which are $x_{1}, x_{2} x_{3}$, and if $A$ arrives at the point $x_{1}^{\prime} x_{2}^{\prime} x_{3}^{\prime}$ as a result of the deformation, then, since by hypothesis the $x_{1} x_{2} x_{3}$ are continuous single-valued functions of the $x_{1} x_{2} x_{3}$ 's, $B$ must also have the co-ordinates $x_{1}^{\prime} x_{2}^{\prime} x_{3}^{\prime}$, after the deformation, i.e. must be at the same point (or infinitely near) A. Consequently, all coincidences remain undisturbed by the deformation (Schlick, 1917a, 181, tr. 1978, I, 241; my emphasis). 
Schlick could plausibly have read the point-coincidence argument as the claim that, since all worlds that agree on such coincidences are equivalent, a choice among them is the result of an arbitrary stipulation. Space assumes a determinate metrical structure only after a decision has been made about the behavior of our measuring instrument. "Poincaré has expressed this tersely in the words: "Space itself is amorphous; only the things in it give it a form" (Schlick, 1917a, 166 , tr. 1978, I, 239, see above on p. 10). Poincaré invited us to declare the bodies to be "rigid" that, when used as measuring rods, lead to the simplest geometry. Schlick argues instead that we should choose "the simplest formulae to express the laws of physics" as a criterion (Schlick, 1917a, 186, tr. 1978, I, 238; my emphasis).

Moving from the consideration of space to that of space-time, it was easy for Schlick to use this sort of argument in the context of general relativity: "Whereas a point transformation in space alone represented ... a change of position and a distortion of bodies, a point-transformation in the four-dimensional universe also signifies a change in the state of motion of the three-dimensional world of bodies: since the time co-ordinate is also affected by the transformation" (Schlick, 1917a, 182, tr. 1978, I, 242; my emphasis). The "relativity of all motions" allegedly proclaimed by general relativity is thus regarded as the consequence of our freedom of deforming space-time as we please; it is simply the four-dimensional counterpart of what Schlick labels "the geometrical relativity of space":

Our considerations about the general relativity of space may immediately be extended to the four-dimensional space-time manifold ... The system of world-lines in this $x_{1}-x_{2}-x_{3}-x_{4}$-manifold represents the happening in time of all events in the world ... If we suppose a complete change of this sort to take place, by which every physical point is transferred to another space-time point in such a way that its new co-ordinates, $x_{1}, x_{2}, x_{3}, x_{4}$, are quite arbitrary (but continuous and single-valued) functions of its previous co-ordinates $x_{1}, x_{2}, x_{3}, x_{4}$ : then the new world is, as in previous cases, not in the slightest degree different from the old one physically, and the whole change is only a transformation to other co-ordinates. For that which we can alone observe by means of our instruments, viz. spacetime-coincidences, remains unaltered. Hence points which coincided at the world-point $x_{1}, x_{2}, x_{3}, x_{4}$ in the one universe would again coincide in the other at the world-point $x_{1}^{\prime}, x_{2}^{\prime}, x_{3}^{\prime}, x_{4}^{\prime}$. Their coincidence - and this is all that we can observe - takes place in the second world precisely as in the first (Schlick, 1917a, 182, tr. 1978, I, 242).

The point-coincidence argument allows Schlick to exorcise the novelty of Einstein's newborn theory by simply inserting it into the context of what was then an already familiar 19th century debate about geometry. In Schlick's reading, the meaning of the requirement that the laws of physics "are 'covariant' for all substitutions" must be found in the fact that "it allows the relativity of space, in the most general sense discussed" (Schlick, 1917a, 182, tr. 1978, I, 238). This is the great achievement of Einstein's theory. Space and time do not have any metric structure given a priori, as Kantians had uncritically assumed; such a structure is introduced only by an arbitrary convention. "In this way," ac- 
cording to Schlick, "Space and Time are deprived of the last vestige of physical objectivity, to use Einstein's words" (Schlick, 1917a, 182, tr. 1978, I, 238).

If one considers the more technical parts of Schlick's paper, it is puzzling how he could find this conclusion philosophically satisfying. Of course, general covariance assures that one is free to introduce any coordination of the physical space that is produced by an arbitrary, if smooth, transformation from the original. However, this transformation must be accompanied by a suitable change of the $g_{\mu \nu}$, so that that the $d s^{2}$ remains unchanged; all measure relations can be "recovered" in the new coordinate system by using the new $g_{\mu \nu}^{\prime}$ to get real distances from coordinate distances. Thus the coordinate transformation is taken into account in general relativity precisely insofar as it does not change the underlying metrical properties. The world, as Hertz had put it, "must be developable onto itself", like the bending without stretching of an inelastic piece of paper (see also Born, 1916, 56), and not freely deformable like a rubber sheet.

This becomes even more evident if one considers that in general relativity as Schlick correctly notices - "the definition of a geodetic line is independent of the co-ordinate system": "the shortest connecting line between two worldpoints, likewise represents a shortest line in the new system of $x_{1}^{\prime}, x_{2}^{\prime}, x_{3}^{\prime}, x_{4}^{\prime \prime}$ " (Schlick, 1917a, 184, tr. 1978, I, 247). For this simple reason the "relativity of all motions" - which was certainly a part of Einstein's heuristic — was not achieved in the final theory. Motion along a geodesic path or deflection from a geodesic path are absolutely different in general relativity, as in previous theories, and no coordinate transformation can eliminate this difference: "the Einstein Law," Schlick continues, "which comprises both inertial and gravitational effects, asserts the world-line of a material point is a geodetic line in the space-time continuum. This laws fulfills the condition of relativity for it is an invariant for any arbitrary transformations, since the geodetic line is defined independently of the system of reference" (Schlick, 1917a, 184, tr. 1978, I, 247). What is "conventional" in Einstein's new theory - a point that Hermann Weyl was probably the first to make completely clear during the 1920 Bad-Nauheim debate (Weyl, 1920) - is the distinction of "inertial and gravitational effects" and certainly not the inertio-gravitional structure itself.

\subsection{The Appendix to the Book Edition of Schlick's 1917 Paper}

As is well known, Einstein was enthusiastic about Schlick's paper (CPAE 8, 297, 389, letter to Schlick of 6 February 1917; Howard, 1984; Hentschel, 1986, cf.), which he read very carefully. He famously corrected Schlick's "infinitesimally flat" interpretation of the equivalence principle (CPAE 8, 314, 417f.; letter to Schlick of 21 March 1917; cf. Norton, 1985). However, no other remarks are made about Schlick's conventionalist reading of the point-coincidence argument. Einstein might still have found it philosophically appealing that general relativity had transformed the rigid "body of reference" of special relativity into a "mollusk of reference", as he had put it in his late 1916 "gemeinverstädiches" book on relativity (published as Einstein, 1917).

However, it is evident that Schlick's reading led him to draw philosophical consequences from the point-coincidence argument that does not seem to have 
much to do with the requirement of general covariance. In the paragraph "Relation to philosophy" — which Schlick added to the book version of his paper (finished May 1917) — Schlick laid down what he later labeled the "method of coincidences", in which Einstein's argument is interpreted as a bridge between physiological space to the abstract mathematical one:

For it is here that experiences arising out of coincidences come into account. In order to fix a point in space, we must in some way or other, directly or indirectly, point to it: we must make the point of a pair of compasses, or a finger, or the intersection of cross-wires, coincide with it (i.e. bring about a time-space coincidence of two elements which are usually apart). Now these coincidences always occur consistently for all the intuitional spaces of the various senses and for various individuals. It is just on account of this that a 'point' is defined which is objective, i.e. independent of individual experiences and valid for all ... Upon close investigation, we find that we arrive at the construction of physical space and time by just this method of coincidences and by no other process. The space-time manifold is neither more nor less than the quintessence of objective elements as defined by this method. The fact of its being a four-dimensional manifold follows from experience in the application of the method itself. This is the outcome of our analysis of the conceptions of space and time ... We see that we encounter just that significance of space and time which Einstein has recognized to be essential and unique for physics, where he has established it in its full right (Schlick, 1917b, 58 , tr. 1978, I, 263).

Schlick again reads Einstein's argument as the claim that coincidences alone are relevant and empirically determinable, and thus the only basis for the construction of the physical space. This interpretation must have sounded natural to the philosophically informed reader. In a 1917 paper, Philip Frank - the successor to Einstein's Prague chair in physics, who would become one of the most important associates of the Vienna Circle - argued that "Einstein joined Mach" by claiming that "space and time properly speaking no longer occurred, but only the coincidence [Koinzidenz] of phenomena" (Frank, 1917, 70, tr. 1950, 73; my emphasis). Mach's name was probably the first to come to mind by interpreting Einstein's appeal to point-coincidences as the only content of a physical theory. Neither could Schlick avoid evoking the Machian flavor of Einstein's remark, even if he rejected the psychologistic reading of the notion of coincidence usually associated with Mach's positivism:

Physics introduces, as its ultimate indefinable conception, the coincidence [Zusammenfallen] of two events; on the other hand, the psycho-genetic analysis of the idea of objective space ends in the conception of the spacetime coincidence [Koinzidenz] of two elements of perception. Are they to be regarded simply as one and the same thing? Rigorous positivism, such as that of Mach, affirms them to be so ... Physics introduces the coinciding [Zusammenfallen] of two events as an ultimate, indefinable concept; but the psycho-genetic analysis of the idea of objective space ends with the concept of the spatiotemporal coincidence [Koinzidenz] of two elements of 
experience. Are both simply the same? Rigorous positivism, such as that of Mach, affirms them to be so. According to him, the directly experienced elements such as colours, tones, pressures, warmths, etc., are the sole reality ... This view is, however, not the only possible interpretation of scientific facts ... [T] he quantities which occur in physical laws do not all indicate 'elements' in Mach's sense. The coincidences which are expressed by the differential equations of physics are not immediately accessible to experience. They do not directly signify a coincidence of sense-data ... We might just as well assume that elements or qualities which cannot be directly experienced also exist. These can likewise be termed 'real' whether they be comparable with intuitional ones or not (Schlick, 1917b, 58-59, tr. 1978, I, 264-265).

Against Mach's positivism, Schlick insists that it is essential to physics to translate the elementary Erlebnisse about coincidences into the abstract framework of a "number manifold" where these relations can be expressed in terms of identity or the neighboring of coordinates values: "We cannot therefore ascribe to physical objects the space of intuition with which our visual perceptions have made us acquainted, nor that which we find present in our tactual presentations, but only a conceptual arrangement, which we then term objective space, and determine by means of a suitably disposed manifold of numbers (co-ordinates)" (Schlick, $1917 b$, , tr. 1978, I, 238). Space-time "in mathematical language" is simply "the manifold of all number quadruples $x_{1}, x_{2}, x_{3}, x_{4}$ " (Schlick, 1917b, , tr. 1978, I, 238). The method of coincidences led us from the physiological space-time as the totality of all possible experience of coincidences to the abstract concept of space-time as the totality of possible quadruples of real numbers.

Schlick was of course proudly convinced that he was exploiting the philosophical implications of Einstein's "public version" of the point-coincidence argument. However, the examples of Schlick's prose we have quoted at length should allow us to see the emperor's new clothes: what Schlick was actually doing was unwittingly rehearsing, without significant changes, Kretschmann's version of the argument as it appears in his 1915 paper. If the emperor was not completely naked, his clothes were surely not new.

Schlick refers to the same authors, Poincare and Mach, and reaches the very same conclusions as Kretschmann: the measure-relations of space are undetermined by experience, which only has access to the set of all possible pointcoincidences, which, in turn, is encoded in the structure of the number-manifold, that is, the set of all possible quadruples of real numbers. Everything else is the result of a stipulation - a stipulation about the behavior of our measuring instruments, clocks and measuring-rods. The only reason to prefer one stipulation over another is due to a criterion of simplicity. The only missing element in Schlick's presentation are Kretschmann's group-theoretical considerations about the relations between invariance and relativity principles. Ironically, as we shall see in the next section, this is exactly the point of view from which Schlick's misunderstanding most clearly emerges (Friedman, 1983, 25ff.; Friedman, 1984, $654)$.

Of course, there is no evidence that Schlick ever read Kretschmann's paper, 
and there is no reason to conjecture that he did. Schlick's "Kretschmannian" reading was, so to speak, an instinctive one. It must have seemed natural at the time to interpret Einstein's point-coincidence remark alongside "the ideas of such men as Mach and Poincaré" (Frank, 1950, 11). After all, this is precisely where the argument comes from, and Schlick was of course acquainted with this tradition before even coming into contact with General Relativity (Engler, 2006).

However, when Einstein "stole" Kretschmann's point-coincidence remark in late 1915, he immediately inserted it into a completely different tradition one that culminated in the work of Ricci and Levi-Civita, and in which general relativity famously represented a "triumph". Considering the argument from this perspective - as Kretschmann himself rapidly came to realize - meant subjecting it to a sort of Kuhnian "Gestalt-switch". In contrast to Kretschmann, however, the logical empiricists were unable to achieve the same change of perspective. They continued to see the duck instead of the rabbit, or, leaving metaphors aside, to see Mach and Poincaré where Ricci and Levi-Civita were meant to be.

\section{Kretschmann after Einstein: The Triviality of the "Public" Point- Coincidence Argument}

Just as Einstein was enthusiastic about Schlick's 1917 paper, he was also pleased with his booklet as well". In a letter to Schlick on 21 May 1917, Einstein described the "last section, 'Relations to Philosophy' as excellent [vortrefflich]" (CPAE 8, Doc. 343, 456). Employing the parable of two different people pursuing physics interdependently, he suggested to Schlick that he should further clarify the notion of what is real in physics: not "the immediately given", but exclusively the "spatiotemporally arranged" (CPAE 8, Doc. 343, 456). The philosophical relevance of these remarks has often been discussed in historical and philosophical literature (Howard, 1984, §1; Howard, 1999, §4). It is, however, rather puzzling that Einstein did not point out to Schlick that his reading of the point-coincidence argument was based on a fundamental misunderstanding.

Just like the early Kretschmann, Schlick claimed that the choice between different space-time geometries endowed with different metrical properties sets of $g_{\mu \nu}$ that cannot be transformed into one another by a simple coordinate transformation - is arbitrary, whereas the "topological" relations represented by point-coincidences are univocally determined by experience. This, however, had nothing to do with Einstein's version of the argument, not even with the "public" version. As, for instance, De Sitter's formulation clearly shows (see above on p. 18), Einstein referred to the possibility of representing the same space-time geometry in different coordinate systems by using different sets of $g_{\mu \nu}$ that differ only by a coordinate transformation. The context in which the argument should be read is not, of course, that of Poincaré's conventionalism colored with a "Machian" flavor, but rather Ricci and Levi-Civita's "absolute differential calculus". 
This is what Kretschmann himself had rapidly come to realize. On 14 August 1917, only several months after the appearance of the first edition of Schlick's booklet, the Annalen der Physik received the manuscript of a second paper of Kretschmann's, which was dated 6 August: Über den physikalischen Sinn der Relativitätspostulate, A. Einsteins neue und seine ursprüngliche Relativitätstheorie (Kretschmann, 1918). The paper — "rediscovered" by James L. Anderson in the mid-1960s (Anderson, 1964, 184; Anderson, 1967, § 10.3) — was destined to become a classic and has therefore been widely discussed in the historical and philosophical literature (Norton, 1995; Rynasiewicz, 1999; Norton, 2003; Pitts, 2008). However, less importance has been given to a comparison between Kretschmann's first and second papers, an issue which will be insisted on here.

As is well known, Kretschmann, after having recalled Einstein's alleged connection between the covariance requirement and relativity principle, famously turned the "public version" of the point-coincidence argument against Einstein himself:

The forms in which different authors have expressed the postulate of the Lorentz-Einstein theory of relativity - and especially the forms in which Einstein has recently expressed his postulate of general relativity - admit the following interpretation (in the case of Einstein, it is required explicitly): A system of physical laws satisfies a relativity postulate if the equations by means of which it is represented are covariant with respect to the group of spatiotemporal coordinate transformations associated with that postulate. If one accepts this interpretation and recalls that, in the final analysis, all physical observations consist in the determination of purely topological relations (["coincidences"] ${ }^{1}$ )) between objects of spatiotemporal perception, from which it follows that no coordinate system is privileged ${ }^{2}$ ) by these observations, then one is forced to the following conclusion: By means of a purely mathematical reformulation of the equations ${ }^{3}$ ) representing the theory, and with, at most, mathematical complications connected with that reformulation, any physical theory can be brought into agreement with any arbitrary relativity postulate, even the most general one, and this without modifying any of its content that can be tested by observation (Kretschmann, 1918, 576; my emphasis; partially transalted in Norton, 1995, 228)

In the footnote 1) Kretschmann refers us to Einstein's 1916 review paper for the choice of "Koinzidenz", instead of his own "Zusammenfallen". Aside from this minor point, Kretschmann clearly recognized his own argument in Einstein's wording. He cites his own 1915 paper in the footnote 2) and thus directs "readers to Einstein's unacknowledged source for his point coincidence argument" (see Norton, 1993 28). However, Kretschmann actually does not claim any priority. He must also have sensed that Einstein had cast his own argument in a very different conceptual framework. In particular, in note 3) Kretschmann adds a reference to Ricci and Levi-Civita's work (Levi-Civita and Ricci-Curbastro, 1900), who were not even mentioned in his first paper.

As is well known, Kretschmann's "triviality charge" against Einstein's requirement of general covariance is based on the idea that special relativity (and 
any other space-time theory) can also be reformulated in a generally covariant way without changing its physical content. "Einstein," Kretschmann points out, based his requirement of general covariance on the fact that all "physical experience ultimately consists in the observation of purely topological relations or "coincidences" between spatiotemporal objects of observation" (Kretschmann, 1918, 577; my emphasis). Consequently, "in experience no reason can be found to privilege any reference system for space and time above all others as the sole legitimate ones" (Kretschmann, 1918). However, Kretschmann points out, this is not a specific feature of general relativity (Kretschmann, 1918).

Kretschmann (Kretschmann, 1918, 579) invites the reader to consider the system of reference $\Sigma\left(x_{1}=x, x_{2}=x, x_{3}=z ; x_{4}=i c t\right)$ where the equations of the light propagation assume the well-known form:

$$
x_{1}-x_{1}^{0}+\ldots+x_{4}-x_{4}^{0}=0
$$

According to Kretschmann we can easily reformulate this equation in a "generally covariant" way, valid for all coordinate systems, by using a quadratic differential form with variable coefficients. In Kretschmann's own notation:

$$
\left\{\begin{array}{l}
\delta \int d s=0 \\
d s^{2}=\sum g_{\mu \nu} d x_{\mu} d x_{\nu}=0 \\
(\lambda \nu, \mu \tau=0)
\end{array} \quad \lambda, \nu, \mu, \tau=1,2,3,4\right.
$$

where $(\lambda \nu, \mu \tau)$ represent the components of the "Einstein's curvature tensor of the space-time manifold" (Kretschmann, 1918, 579) in the old-fashioned notation (Kretschmann refers us to Christoffel, 1869). Let us cover the Minkowski space-time with a rectangular grid of coordinates; then of course $g_{\mu \nu}=1$ for $(\mu=\nu)$ and $g_{\mu \nu}=0$ for $(\mu \neq \nu)$. If we perform a smooth substitution of the independent variables $x_{\nu}$, according to the well-known formula of the absolute differential calculus, in place of the original $g_{\mu \nu}\left(x_{\nu}\right)$ we get a new non-standard system $g_{\mu \nu}^{\prime}\left(x_{\nu}^{\prime}\right)$ under the condition that $d s^{2}$ remains invariant. For instance, one can introduce polar coordinates (Kretschmann, 1918, 578); geodesics become curved lines or, more precisely, since the coordinates are curved, geodesic lines are not simple linear functions of the coordinates. However, this is merely a mathematical difference. The Riemann-Christoffel-Tensor $(\lambda \nu, \mu \tau)$ necessarily vanishes in both cases. Thus it is always possible to find a coordinate system in which the $g_{\mu \nu}$ assume their standard Minkowski values. The difference of the values of $g_{\mu \nu}$ is only an artifact introduced by the mathematical formalism and does not imply any "real" physical difference.

Kretschmann could then show that it is very easy to provide a generally covariant formulation of special relativity without making the theory more "relativistic". One can still recognize the Lorentz group as the one that is more adherent to the physical content of the theory; it is the only group that would preserve the equation (1) intact, whereas the passage from cartesian to polar coordinates does not. The requirement of general covariance therefore has nothing to do with an extension of the relativity principle: 
According to the general version of a relativity postulate we have provided above, the same laws for light-propagation, depending on the form of their presentation, satisfies - once represented by (2) - the most general relativity postulate, and in the other form, (1) only a special relativity postulate. The same would apply to all physical laws. In fact according to the investigations of Ricci and Levi-Cività [sic] it may scarcely be doubted that one can bring any physical system of equations into a generally covariant form without alteration of its observationally testable content. This is obvious from the beginning, if one once again recalls that strictly only purely topological facts of natural phenomena or, according to Einstein, coincides are observable (Kretschmann, 1918, 579, my emphasis).

Although the reference to the "topological facts" in which all coordinate systems agree recalls Kretschmann's wording in his 1915 paper, the problem Kretschmann is dealing with has become completely different. As we have seen, in 1915 Kretschmann, inspired by Mach and Poincaré, was concerned by the fact that the choice between different relativity theories, characterized for instance by different laws for the propagation of light - e.g. Galilei-Hertz vs. Lorentz-Einstein — is only conventional. In 1917 Kretschmann, relying on Ricci and Levi-Civita's investigations, wanted to show that the very same Lorentz-Einstein theory can be presented in arbitrary coordinate systems without changing its physical content: "the light-propagation equation of the original theory of relativity could be put into generally covariant form" by merely introducing "the undetermined coefficients $g_{\mu \nu}$ into the expression for the line element" (Kretschmann, 1918, $585-6)$

The very possibility of formulating special relativity in a generally covariant form, according to Kretschmann, shows the difference between the principle of relativity as a physical principle and the covariance of the equations as a mathematical requirement. Roughly, Kretschmann identifies the relativity principle with the existence of a 10-parameter continuous group of transformations, which leaves unchanged the expression for the four-dimensional distance between two points $d s^{2}$. In a flat Minkowski space-time, the Lorentz-transformations leave unchanged the values $g_{\mu \nu}=1,1,1-1(\mu=\nu)$ and $g_{\mu \nu}=0(\mu \neq \nu)$, which thus remain the same functions of the coordinates. Geometrically (§§23-25) this means that such transformations "map back onto themselves [in sich selbst überführen]" (Kretschmann, 1918, 583) all geodesic paths of light rays $\delta \int d s^{2}=0$ and free-falling particles $\leq 0$ - i.e. all possible inertial paths.

On the contrary, in a non-uniform, general-relativistic space-time there are, in the general case, no transformations that leave the $g_{\mu \nu}$ unchanged; in general they are different functions of the coordinates. Kretschmann showed that if two sets of $g_{\mu \nu}$ determine not only the same light rays' trajectories, but also the same path of free-falling particles, then those metrics differ at most by a position-independent constant $\lambda .{ }^{5}$ However, this degree of freedom can also

\footnotetext{
${ }^{5}$ This turnout to be a groundbreaking result that will be developed by Weyl 1921; Lorentz 1923; cf. Ehlers et al., 1972 for a modern treatment)
} 
be eliminated by cosmological considerations: the "curvature character of the world" [Krümmungscharakter der Welt]" (Kretschmann, 1918, 591, n. 2) forced us to assume a value of $\lambda$, so that the curvature radius is $=1$.

No trace of conventionalism seems to have survived in Kretschmann's epistemology. On the contrary, he now emphasizes that general relativistic space-time has a well-defined, although variable metrical structure. In particular (§§17-21), Kretschmann was able to prove that, in matter- and field-free regions of spacetime - where the Einstein tensor $B_{\mu \nu} \equiv \sum_{\mu \nu}(\lambda \nu, \mu \tau) g^{\lambda \tau}=0-$, there might still be a set of four non-vanishing components of the Riemann-Christoffel tensor $(\lambda \nu, \mu \tau)$ - the only nontrivial scalars that can be constructed purely from the Riemann tensor - which, in the general case, are different from point to point ${ }^{6}$. In the vast majority of space-times that satisfy the gravitational field equations, there is no transformation that maps such scalars back onto themselves besides identity.

The conclusion that Kretschmann drew from these considerations has become famous: "Einstein's theory satisfies no relativity principle at all ... it is a completely absolute theory" (Kretschmann, 1918, 610). As Kretschmann showed in the final paragraphs of the paper ( $\S 27-29)$, in order to satisfy a "general relativity principle", a theory would have to admit that world-lines can "be mapped back into themselves by an arbitrary continuous deformation [Verzerrung]" (Kretschmann, 1918, 612). This would, however, destroy the difference between geodesic and non-geodesic paths, making a theory based on the "geodesic hypothesis" - small 'freely-falling' bodies move along geodesic trajectories - completely senseless. Actually, as Kretschmann proves, special relativity has the largest relativity group on "a space-time manifold with Minkowski normal form of the line element" (Kretschmann, 1918, 610). In Minkowski space-time there is no theory "more relativistic" than special relativity.

Ironically, if Kretschmann's first 1915 version of the point-coincidences argument had de facto anticipated all the essential lines of Schlick's interpretation of general relativity, Kretschmann's second 1917 formulation of the argument already definitively refuted it. Adopting Kretschmann's group-theoretical point of view on the relativity principles, it is easy to see where Schlick's mistake is located. By following Einstein's claim, with some understandable ingenuity, that the general covariance of his theory embodied an extension of the principle of relativity to acceleration (cf. for instance Norton, 1995), Schlick believed that in general relativity the laws of physics are "invariant" with respect to a largest group of transformations, which preserve only the relations of coincidence and neighborhood between world-points (Friedman, 1983, 25ff.). As we now know, the exact opposite is true - the invariance group of general relativity is, in general, the identity group.

\footnotetext{
${ }^{6}$ This is another major achievement of Kretschmann's paper that will be later rediscovered by Komar, 1958; cf. below on p. 38
} 


\section{Einstein after Kretschmann Once Again: Countering Kretschmann's Objection}

At the time, the correct relation between covariance and invariance was of course not so easy to grasp (Norton, 1995). On 1. February 1918 Einstein could still write to Sommerfeld that "Schlick's presentation is masterful [meisterhaft]" (CPAE Doc. 453, 627). On the very same day, the Heft of the Annalen der Physik that contained Kretschmann's paper was distributed. In a letter from 17 February 1918 (CPAE 8, Doc. 465, 649f.), Gustav Mie must have drawn Einstein's attention to Kretschmann's objection. Mie - who was in correspondence with Kretschmann - emphasized in particular that a theory satisfying a general principle of relativity would have looked exactly like Kretschmann described it in the last paragraphs of his paper. Einstein famously replied to "the penetrating work of Kretschmann [scharfssinige Arbeit von Kretschmann]" (Einstein, 1918, 241) immediately thereafter in an article received by the $A n$ nalen der Physik on 6 March 1918 (Einstein, 1918).

Einstein regarded "Hr. Kretschmann's argument" as correct: "Since the totality of physical experience refers only to coincidences," he summarizes, "it must always be possible to represent experiences concerning the lawful dependencies of these coincidences by means of generally covariant equations." (Einstein, 1918, 242). Einstein nevertheless emphasized that the link between the principle of relativity, the requirement of general covariance and the pointcoincidence argument possesses "an important heuristic force", if combined with a principle of simplicity (Einstein, 1918, 242). A generally covariant formulation of pre-general-relativistic physics would be unnecessarily complicated "from the standpoint of the absolute differential calculus" (Einstein, 1918, 242; cf. Bergmann, 1942, 159).

Einstein's answer to Kretschmann's objection — published on 24 May 1918 - is not usually considered to be particularly effective, but it reconfirms the "standpoint" from which the argument should be understood. A more compelling counter-objection may be found, at least indirectly, in the first edition of Hermann Weyl's Raum-Zeit-Materie (Weyl, 1918; the book was prepared from the lecture notes of a course given in the summer term of 1917 at ETH Zurich), the proofs of which Einstein had started to read with "real enthusiasm" at the beginning of march 1918 (CPAE 8, Doc. 472 and Doc. 476).

Weyl - without mentioning Kretschmann - also emphasizes that the requirement of general covariance is a "purely mathematical process [rein mathematische Angelegenheit]". By letting the $g_{i k}$ appear in the expression of the line element, "we may formulate physical laws so that they remain invariant for arbitrary transformations; this is a possibility that is purely mathematical in essence and denotes no particular peculiarity of these laws" (Weyl, 1918, 181; tr. 1922, 226). However, whereas in special relativity the $g_{i k}$ display the preassigned Malinowski-values, in general relativity the $g_{i k}$ have become "physical field quantities" - just like "the components $\phi_{i}$ of the electromagnetic potentials" - "to which there corresponds something real": "This assumption, rather than the postulate of general invariance, seems to the author to be the real pivot 
of the general theory of relativity" (Weyl, 1918, 181; tr. 1922, 227).

In the Encyclopedia article on relativity theory, which the prodigiously young Wolfgang Pauli had completed in December 1920 (cf. Pauli, 1921, 175; the article was published on 15 November 1921), Weyl's approach is explicitly used against Kretschmann's objection (Pauli, 1921, 711; cfr. Dieks, 2006, 186f.). There is, however, a profound difference between general relativity and traditional field theories. In general relativity, "once we have found any kind of solutions of the general covariant field equations, we can derive from them an arbitrary number of other solutions by means of a different choice of coordinates" (Pauli, 1921, 192; tr, 1981, tr. 160). For this reason, as Pauli notices, "Einstein had, for a time, held the erroneous view that one could deduce from the non-uniqueness of the solution that the gravitational equations could not be generally covariant" (Pauli, 1921, 192; tr, 1981, tr. 160). In footnote 315 Pauli gives credit to Hilbert for having been the first to show that "the contradiction with the causality principle is only apparent": "the many possible solutions of the field equations are only formally different. Physically they are completely equivalent" (Pauli, 1921, 192; tr, 1981, tr. 160).

As De Sitter had clearly recognized (and in a published writing; see above on p. 18), the claim that only coincidences, and not their coordinates, are observable was precisely Einstein's way of dealing with such a surplus of mathematical structure that has no correlate in physical reality. Thus Einstein's remark does not serve at all, as Schlick thought, to deprive every mathematical structure that is not reducible to point-coincidences of physical significance. In the 1920s Schlick was also in personal and epistolary contact with Pauli (who at that time was a strict positivist, like his godfather Mach; Meyenn, 2009, §5). Schlick, however, was never able to appreciate the philosophical relevance of the presence of such redundancy in the mathematical formalism of general relativity: the same physical world can be described by mathematically different solutions of the field equations.

\section{Back to Kretschmann: The Unacknowledged Triumph of Kretschmann's Conventionalist Epistemology}

\subsection{Logical Empiricists and Kretschmann's Early Point-Coincidence Argument}

In his 1918 Allgemeine Erkenntislehre, Schlick further articulated the epistemological consequences of his "method of coincidences" (Schlick, 1918, 236) by introducing a form of "critical realism" — probably inspired by Riehl's ${ }^{7}$ work (Heidelberger, 2007). "Coincidences" — on which different senses of the same and different individuals necessarily agree — give access to nothing less than the things-in-themselves. This rather surprising "realistic" conclusion became the philosophical counterpart to the conventionalist reading of general relativity that remained unchanged in the successive editions of his 1917 booklet (Schlick,

\footnotetext{
${ }^{7}$ As we have mentioned on p. on page 9 also Kretschmann had attended Riehl's lectures in Berlin.
} 
1919, 1920). Schlick, needless to say, was convinced that he was exploiting the philosophical implications of Einstein's 1916 point-coincidence argument. What he was actually doing - as we have seen — was rehearsing Kretschmann's version of the argument as it appears in a minor article from 1915, in an irony of fate, before general relativity as we know it even existed. The conventionalist interpretation of general relativity was, so to speak, born dead. Kretschmann's second and more famous 1917/1918 paper should have put Schlick on the right track. However, as we shall see, the paper went unnoticed in the philosophical community.

As the young Reichenbach had seen with impressive lucidity in his first monograph (Reichenbach, 1920/1965), general covariance implies the "relativity of coordinates" and not the "relativity of geometry", not the freedom of choosing among different geometries, but the freedom of expressing the same geometry in different coordinate systems. As Reichenbach had rightly put it, it is true that "the four space-time coordinates can be chosen arbitrarily, but that the ten metric functions $g_{\mu \nu}$ may not be assumed arbitrarily; they have definite values for every choice of coordinates" (Reichenbach, 1920, 86; tr. 1965, 90). As we just mentioned, the $g_{\mu \nu}$ are univocally determined by field equations, but of course only up to four arbitrary functions. However, "this indeterminacy [Unbestimmtheit]" (Reichenbach, 1920, 96-97; tr. 1965, 102; my emphasis) does not reflect any lack of univocality of the geometrical structure of the world: "There exist dependency relations among the metric coefficients, and if four of them are arbitrarily given for the whole space, then the other six are determined by transformation formulas" (Reichenbach, 1920, 96-97; tr. 1965, 102; my emphasis). Even if Reichenbach did not provide further details, as we have seen, the presence of such "redundancy" in the mathematical apparatus was precisely the "philosophical" problem that the point-coincidence argument was meant to solve: solutions of the field equations that differ only by a coordinate transformation, as Weyl put it, "represent the same objective course of the world" (Weyl, 1918, 190; tr. 1922, 237).

As is well known, Reichenbach's promising line of reasoning unfortunately did not survive the exchange of a few letters with Schlick (on this topic see Parrini, 2005). The "conventionalist" interpretation of the point-coincidence argument rapidly became the "official" reading defended by early Logical Empiricism. In the commentaries to Helmholtz's epistemological writings, which Schlick edited with Paul Hertz ${ }^{8}$ in 1921 (Helmholtz, 1921), he could attempt to interpret even Helmholtz's celebrated convex-mirror thought experiment (Helmholtz, 1870/1883) as a precursor to Einstein's point-coincidence argument. Whatever pairs of objects are congruent in our world must be congruent in its distorted mirror image, Helmholtz had argued, and no decision can be made about which one is the undistorted world (cf. DiSalle, 2006, 82ff.). "Helmholtz' proposition - Schlick comments - can therefore be extended to the truth that no occurrences whatsoever can be ascertained physically other than meetings of points,

\footnotetext{
${ }^{8}$ Of course the same Paul Hertz we have mentioned earlier on p. 7
} 
and from this Einstein has logically drawn the conclusion that all physical laws should contain only statements about such coincidences" (Schlick's commentary in Helmholtz, 1921, 30, n. 39; tr. 1977, 34). Without any other sources of information, one must be content considering the undistorted world as the one in which "much simpler mechanics prevails" (Schlick's commentary in Helmholtz, 1921, 34, n. 46; tr. 1977, 35).

The impression that this is nothing more than the 1915 "Kretschmannian" version of the point-coincidence argument becomes hard to resist by reading Rudolph Carnap's dissertation, finished before February 1921 under the supervision of the neo-Kantian Bruno Bauch (Gabriel, 2004). Carnap made one last step in philosophers' unwitting re-appropriation of Kretschmann's argument by explicitly labeling the point-coincidences as "topological" properties, a parlance that would become standard among the logical empiricists in the following years: "Only space-time encounter ("coincidence") [Zusammenfallen ("Koinzidenz")] is physically established [feststellbar] ... Hence only topological determinations are univocal [eindeutig]" (Carnap, 1922, 83; note to page 39ff.). Carnap of course mentions Einstein's 1916 review paper (Einstein, 1916) among his sources (together with Schlick, 1920). Interestingly, he also mentions the positivist Joseph Petztold, who expressly linked Einstein's language of coincidences and Mach's reduction of scientific knowledge to "coincidences of perceptions" (Petzoldt, 1921, 64). Of course, a reference to Poincaré here (Poincaré, 1906b) could not be lacking (Carnap, 1922, 83; note to page 39ff.)

If Carnap sought to illuminate the meaning of Einstein's point-coincidence argument by resorting, directly or indirectly, to Mach and Poincaré's authority, it is all but surprising that he ended up producing the exact replica of Kretschmann's 1915 line of reasoning: "the content [Inhalt $]$ of the experiential statement is unchangeable (invariant) under one-to-one continuous space mappings (transformations)", but "this holds for topological statements and for these only"; non-topological determinations, e.g. projective and metrical relations, "do not remain unchanged against those space mappings. They are therefore not pure statements of a factual basis, but depend on form determined by choice [von wahlbestimmter Form]" (Carnap, 1922, 39).

If one puts aside Carnap's still "Kantian" framework (Mormann, 2009), this rapidly becomes the logical empiricists' customary reading of Einstein's general covariance requirement. In his 1921-1922 review article, Der gegenwärtige Stand der Relativitätsdiskussion, Reichenbach explains that when "Einstein established the general theory, he regarded the fact that, in principle, only coincidences of physical things are observable as an essential premise". General relativity - Reichenbach continues - "considers coincidences as the only invariants, and relativizes merely the metrical relations between the coincidences" (Reichenbach, 1922a, 332, tr. 1978, I, 15). This remark - inserted in passing in the context of a polemic against Josef Petzold's "machian" reading of the notion of coincidence (Petzoldt, 1921, 64) - clearly shows Reichenbach now took for granted that in general relativity the "metrical relations between the coincidences" are not preserved by a general coordinate transformation (Reichenbach, 1922a, 332, tr. 1978, I, 15). 
Reichenbach (unfortunately) had come to reject his previous claim that "only with regard to the arbitrariness of the co-ordinates is space an ideal structure; the metric, however, expresses an objective property of reality" (Reichenbach, 1922a, 356; tr. 1878, I, 34f.; my emphasis). He now claims that "[t]his conception does not contradict conventionalism". Since "a metric emerges only after the physical laws have been established" - he writes - then "one can also change the metric, provided one changes the laws of physics correspondingly. But the relation between these modifications expresses an invariant fact" (Reichenbach, 1922a, 356; tr. 1878, I, 34f.; my emphasis) which, of course, is represented precisely by the infamous point-coincidences.

Kretschmann's 1917/1918 paper should have made clear that the pointcoincidence argument should actually be read in the context of the work of Ricci and Levi-Civita: transformations preserving coincidences are considered in as much as they do not change the metric, but only its mathematical expression in a different coordinate system. However, Kretschmann's second paper had gone nearly unnoticed. In a letter to Reichenbach on 27 March 1922, Einstein - by sending the drafts of his review article back to Reichenbach specifically lamented the absence of any reference to Kretschmann in the paper. But Kretschmann, who in 1920 had become Privatdozent in Königsberg (Habe, 1955, 647) — was in fact "highly esteemed by Einstein" (Arnold Sommerfeld to Henry A. Erikson 13. Oktober 1922). ${ }^{9}$

On 2 May, Reichenbach wrote to Schlick: "Einstein wrote me that I should also have mentioned Kretzschmann [sic]". But he openly admitted: "I am however not very clear about the meaning of Kretzschmann's [sic] objection and must first still correspond with Einstein about that" (Schlick and Reichenbach, 1920-22). We do not know if this exchange ever took place. However, Kretschmann's second paper would have clearly showed the misunderstanding behind the metrical conventionalism that Reichenbach had by that time fully implemented in his philosophical writings (Reichenbach, 1922b): general covariance does not imply at all that we can choose any kind of geometry we please by changing the physical laws. What it does imply is that we can express the very same physical laws by using any kind of coordinate system we please.

\subsubsection{Logical Empiricism and Kretschmann's Conventionalism}

If our reconstruction is correct, we have to confront an uncomfortable but simple truth: the early logical empiricists misread Einstein's 1916 point-coincidence argument as though it were Kretschmann's 1915 point-coincidence argument. Consequently, they were led to believe that the essential feature of general relativity is that the metric relations - the lengths of world-lines - do not remain invariant under a deformation of space-time that preserves point-coincidences. The mistake of this reasoning is easy to glean, and is rather crude. As Arthur Stanley Eddington famously put it, general-relativistic space-time "is not a lot

\footnotetext{
${ }^{9}$ Deutsches Museum München, Archiv, Archiv NL 89, 019, Mappe 4,1; http://sommerfeld.userweb.mwn.de/KurzFass/03996.html)
} 
of points close together, it is a lot of distances interlocked" (Eddington, 1923, 10). Distances do remain invariant under a transformation that is accompanied by the suitable change of the coefficients $g_{\mu \nu}$. Of course, this is the relevant point of the requirement of general covariance. It is precisely why Kretschmann, in 1917, did not attribute any physical content to this requirement.

Having failed to recognize that Einstein had inserted Kretschmann's language of coincidences into a different tradition, it is after all not surprising that the logical empiricists ended up repeating Kretschmann's early epistemological reflections. Curiously, this did not escape the attention of some of their contemporaries. Oskar Becker, in his 1922 Habilitationsschrift, Beiträge zur phänomenologischen Begründung der Geometrie und ihrer physikalischen Anwendungen - published on Jahrbuch für Philosophie und phänomenologische Forschung in 1923, when he became Husserl's assistant — presented Kretschmann's 1915 paper as the clearest expression of conventionalism:

In order to characterize more precisely this view [conventionalism], we give here an overview of Kretschmann's work. Each measurement of spatiotemporal quantities is carried out by bringing the measuring instrument into superposition with parts of the object to be measured. Only the coincidence [Zusammenfallen] of parts of the measuring instrument with parts of the object measured can be observed; these are purely topological relations between two spatiotemporal objects (§ 1). - Accordingly all topologically identical mappings [Abbildungen] of the phenomenal world are equivalent [gleichberechtigt] (§ 4). The spatiotemporal reference-system of physics is a four-dimensional number-manifold with undetermined (!) metrical structure [Maßbestimmung] (§ 5). Through observations and the mapping-postulate alone no specific measure-determinations [Maßbestimmungen] between spatiotemporal coordinates are given. ... Nontopological, kinematic properties of a physical system of laws are mere conventions (!).

In this way the non-topological physical statements do not lose their scientific value. They are downgraded to formulations of topological laws valid for the "economy of thought". This however has downright devastating consequences for the very content [Bestand] of theoretical physics! (Becker, 1923, 525).

Becker's indignant rejection of Kretschmann's conventionalism - emphatically laden with exclamation marks — was clearly inspired by Weyl (with whom Becker was in correspondence, cf.: Mancosu and Ryckman, 2002, 2005). In those years, Weyl, more than anyone else, had insisted on the "devastating consequences" of reducing — as Schlick actually did — the "four-dimensional world" to an arbitrarily deformable "mass of plasticine" (Weyl, 1924, 198). The philosophical achievement of general relativity certainly does not consist in having discovered the conventionality of every non-topological space-time structure, but instead in having transformed it into a "structure-field [Strukturfeld]" (Weyl, 1931, 338), which is just as real as the electromagnetic field (Weyl, 1924, 198).

Unfortunately, it was precisely the "Kretschmannian" conventionalism described by Becker that became the official logical-empiricist philosophy of space 
and time. In his 1924 Axiomatik der relativistischen Raum-Zeit-Lehre (Reichenbach, 1924), Reichenbach, after again having rejected the positivistic blurring of subjective and objective coincidences (Reichenbach, 1924, 14; tr. 1965, 19), explicitly maintains that "space and time in the General Theory of Relativity mean the same as in the special theory although without any metric" (Reichenbach, 1924, 155; tr. 1965, 195; my emphasis); the "topological properties turn out to be more constant that the metrical one", so that Reichenbach famously argues that "the transition from the special theory to the general one represents merely a renunciation of metrical characteristics, while the fundamental topological character of space and time remains the same" (Reichenbach, 1924, 155; tr. 1965, 195).

Without underestimating the substantial divergences between Schlick's Vienna Circle and the emerging Berlin group gathered around Reichenbach, a common tendency can at least be recognized in the philosophy of space and time (cf. Ryckman, 2007, 200-207): if Schlick still defended his "method of coincidences" in the second expanded edition of his Allgemeine Erkenntnislehre (Schlick, 1925), Carnap, in his first post-doctoral writings (Carnap, 1923, 1925), could easily translate his early "Kantian" conventionalism into an empiricist framework (Carnap, 1922).

Reichenbach's monograph, Philosophie der Raum-Zeit-Lehre (Reichenbach, 1928) - which, as a letter from Reichenbach to Schlick (cited in Schlick, 2006, vol. 6, 175) reveals, was already finished in 1926 - classically summed up the logical empiricists' reading of general relativity in the well-known theories of "equivalent descriptions" and the "relativity of geometry": we are free to choose among topologically equivalent, but physically different metric spaces that can be smoothly deformed into one another by universal "forces" (such as gravitation) "preserving coincidences" (Reichenbach, 1928, ; tr. 1958, 27). Reichenbach's line of reasoning is well summarized by the following passage "The coordinate system assigns to the system of coincidences, of point-events, a mutual order that is independent of any metric. This order of coincidences must therefore be understood as an ultimate fact" (Reichenbach, 1928, 337; tr. 1958, 287; my emphasis).

In 1926 Reichenbach obtained a teaching position in "natural philosophy" at the University of Berlin (Hecht and Hoffmann, 1982) - thanks to Einstein's intervention. In the same year Kretschmann became professor extraordinarius at the University of Königsberg (Habe, 1955, 647). Although in the meantime he had published other technical works on relativity (e.g. Kretschmann, 1920, 1924), his interests seem to have moved to quantum mechanics (see for instance his 1929 popular lecture published as Kretschmann, 1930). However, he does not seem to have noticed the enormous success that his point-coincidence argument - although under false pretenses - enjoyed in the philosophical community.

Karl Popper's 1929 Axiome, Definitionen und Postulate der Geometrie (Popper, 1929) - a scholarly survey on the state of the art in philosophy of geometry - disappointingly shows that few steps had been made by philosophers of science beyond Kretschmann's first version of the argument: 
The possibility of interpreting the experience differently is based on the fact that the metric [Maßsetzung] or the the type of geometry [Geometrieart] can be chosen freely.

Even in the non-metrical projective geometry there is a moment that is completely analogous to that of the determination of the metric [Maßsetzung] ...

The case of the topology is different. Here every metrical moment is completely turned off [ausgeschaltet]. There is therefore no way to interpret the experience.

This is the reason why the topological finding [topologische Befund] can truly be considered as the finding [Befund] of the corresponding [jeweiligen] uninterpreted "facts of the spatial experience" [des ... Tatbestandes der räumlichen Erfahrung] ...

The propositions of applied topology must therefore be regarded as empirical propositions valid (without any further limitation) a posteriori.

The relationship between space points that is relevant to topology is that of the infinitesimal neighborhood or coincidence.

The coincidence of space-elements is the only distinctive [ausgezeichnete] uninterpreted geometrical fact of experience that can exist between spaceelements (It follows that the notion of coincidence [Koinzidenzbegriff] has special epistemological significance for a possible physics that proceeds as a hypothesis-free, strictly empirical physics). (Popper 1929, now in Popper, 2006, 376f.)

If this passage provides a good summary of Schlick, Reichenbach, and Carnap's epistemology of geometry - from whose writing the young Popper abundantly draws - then the mature philosophy of space and time of perhaps the most influential movements in the history of philosophy of science appears to be disarmingly unimpressive. Nothing significant has been added, and no more sophisticated philosophical consequence has been drawn than what a young Ph.D. student of Planck's had suggested more than a decade earlier.

\section{Conclusion}

After the war, Kretschmann continued his career in East Germany as a professor of theoretical physics at the Martin-Luther University of Halle-Wittenberg (Habe, 1955). Most of the European adherents of Logical Empiricism had moved to the United States in the second half of the 1930s, and had essentially abandoned their interest in relativity.

In his famous contribution (Reichenbach, 1949) to the Library of Living Philosopher's volume in Einstein's honor (Schilpp, 1949), Reichenbach still regarded the theory of relativity's most significant achievement as the "discovery" that space-time has no intrinsic metrical structure, but only a topological one (Reichenbach, 1949). It has of course become an easy move to refute this reading by resorting to Einstein's last pronouncement about his own theory, which was published shortly thereafter: if we imagine the metric structure to be removed - Einstein famously claimed - there remains "absolutely nothing, and also no 'topological space"' (Einstein, 1952, 155). 
As we have tried to show, one of the main reasons for this substantial failure must be located in the logical-empiricists' irresistible inclination to read Einstein's point-coincidence argument in the form Kretschmann had originally formulated it. Whereas Einstein had taken nothing from Kretschmann but the expression "point-coincidences", the logical empiricists instinctively dragged along with it the entire apparatus of Kretschmann's conventionalism.

The logical empiricists probably never stumbled onto Kretschmann's paper; they simply drew from the same philosophical tradition Kretschmann was familiar with, a tradition dominated by authors such as Poincaré and Mach. Einstein's coincidence parlance, then, appeared to provide convincing evidence for considering general relativity as the last heir of such a tradition (cf. Frank, 1949); it was the crowning moment of a process that progressively impoverished physically significant mathematical structure, which was finally reduced to the topological or manifold structure of number quadruples, that is, the space-time coincidences. It is impossible to make an empirically founded choice between physically different geometries that agree on such coincidences, which is the only structure that experience can univocally determine. Carnap's 1956 preface to the English translation of Reichenbach's monograph (Reichenbach, 1958) shows that he still considers this to be the main achievement of the book (cf. also Martin Gardner's typescript of Carnap's 1958 seminar on the foundation of physics published as Carnap, 1966).

As we have seen, however, if Einstein did deftly steal the point-coincidence argument from Kretschmann's paper, he used it to solve a problem that emerged from a completely different tradition: one which came from the work of Riemann and Christoffel and culminated in Ricci and Levi-Civita's classic 1900 paper (Levi-Civita and Ricci-Curbastro, 1900). By inserting Kretschmann's turn of phrase into this context, Einstein was led to a somehow specular result: different solutions of the field equations that differ only by a coordinate transformation appear mathematically distinct, but actually represent the very same physical situation, since they agree on point coincidences, which are the only observables.

It was Peter Bergmann - Einstein's assistant at Princeton since 1936 and his school, who, in the mid-1950s, re-discovered a notion of "coincidence" that was more akin to Einstein's, by discussing the problem of 'true observables' in general relativity (Bergmann, 1956). General covariance - as Bergmann's post-doc student Arthur Komar put it - forces us to accept that "the potentials of the gravitational field, as a function of these coordinates" are not observable. Consequently, "[g]iven two metric tensor fields, one cannot readily tell whether they represent two distinct physical situations or whether they represent the same physical situation but in two different coordinate systems" (Komar, 1958, 1182; my emphasis).

In order to "remove the ambiguity engendered by general covariance" (Komar, 1958, 1182), Komar suggested using the four non-vanishing invariants of the Riemann tensor in empty space-times to individuate the points of space-time (cf. Stachel, 1993, for a more recent appraisal of this method). In the generic case, where no symmetries are present, the four scalars $A^{i}$ that "one is able to construct by combining the metric tensor, the Riemann tensor, and its covari- 
ant derivative, will in general have different values at different world points" (Komar, 1958, 1182). The value of the gravitational potentials $\bar{g}^{i k}$ at a certain world-point where the coordinates $x^{i}$ have certain values is not an observable, for the identification of a world-point by means of coordinate values depends on the choice of coordinates. On the contrary, the value of the potentials $\bar{g}^{i k}$ at a certain world-point where the four non-vanishing invariants of the Riemann tensor $A^{i}$ have certain values is an observable, since this information does not depend on the choice of coordinates (Komar, 1958, 1183).

Such "Komar events" (Earman, 2002, §5) must then be regarded as the natural heir of Einstein's notion of "coincidence" (Bergmann, 1961), namely, the coincidences of the values of the metric tensor field $\bar{g}^{i k}$ and of the scalar field $A^{i}$ (Bergmann and Komar, 1960; cf. Bergmann, 1968, §§21 and 23 for a popular presentation). Curiously, this notion of coincidence also bears a subtle relation to Kretschmann's work. As we have briefly mentioned (see above 6 ), Kretschmann was the first to resort to four invariants of the Riemann tensor to individuate the points of space-time with invariant labels (the so called Kretschmann-Komar coordinates). It is of course this notion of coincidence that has become relevant in the modern debate.

General relativity does reveal the existence of a "class of equivalent descriptions" (Reichenbach, 1951) of different physical situations, that is, different possible physical geometries that agree only in coincidences, as Reichenbach who was curiously in contact with Einstein's other Princeton assistant, Valentine Bargmann (cf. Reichenbach, 1944, vii) — believed. On the contrary, general covariance has a physical meaning in as much as it shows, as Bergmann put it, that there is an "equivalence class of solutions" that describes the "same physical situation" (Bergmann, 1961), since they agree on coincidences.

As is well known, in the current coordinate-free parlance this means that general-relativistic space-time is "not just one pair $(\mathcal{M}, g)$ but a whole equivalence class of all pairs $\left(\mathcal{M}^{\prime}, g^{\prime}\right)$ which are equivalent to $(\mathcal{M}, g)$ " (Hawking and Ellis, 1973, 56). It was precisely the worrisome presence of these redundant, non-physical degrees of freedom that Einstein was able to exorcize by resorting to Kretschmann's turn of phrase. A space-time corresponds to a gravitational field; but a gravitational field corresponds to an equivalence class of space-times. "This is the true significance of the concept of general covariance, as Peter Bergmann among others has long known" (Stachel, 1987, 203).

\section{References}

Anderson, J. L., 1964. Relativity Principles and the Role of Coordinates in Physics. In: Chiu, H.-Y., Hoffmann, W. F. (Eds.), Gravitation and Relativity. W.A. Benjamin, Inc., New York, pp. 175-194.

Anderson, J. L., 1967. Principles of Relativity Physics. Acad. Pr., New York [et al.].

Banks, E., 2003. Ernst Mach's World Elements: A Study in Natural Philosophy. Springer, Dordrecht. 
Becker, O., 1923. Beiträge zur phänomenologischen Begründung der Geometrie und ihrer physikalischen Anwendung. Jahrbuch für Philosophie und phänomenologische Forschung 6, 385-560, republished as Becker, 1973.

Becker, O., 1973. Beiträge zur phänomenologischen Begründung der Geometrie und ihrer physikalischen Anwendung, 2nd Edition. Niemeyer, Tübingen, unchanged new edition of Becker, 1923.

Bergmann, P. G., 1942. Introduction to the Theory of Relativity. Prentice-Hall, Inc., New York.

Bergmann, P. G., 1956. Fifty Years of Relativity. Science 123 (3195), 487-494.

Bergmann, P. G., 1961. Observables in General Relativity. Reviews of Modern Physics 33 (4), $510-514$.

Bergmann, P. G., 1968. The Riddle of Gravitation. Scribner, New York.

Bergmann, P. G., Komar, A. B., 1960. Poisson Brackets Between Locally Defined Observables in General Relativity. Phys. Rev. Lett. 4, 432-433.

Born, M., 1916. Einsteins Theorie der Gravitation und der allgemeinen Relativität. Physikalische Zeitschrift 17, 51-59.

Brading, K., Ryckman, T., 2008. Hilbert's 'Foundations of Physics': Gravitation and electromagnetism within the axiomatic method. Studies in History and Philosophy of Modern Physics 39, 102-153.

Carnap, R., 1922. Der Raum. Ein Beitrag zur Wissenschaftslehre. Reuther \& Reichard, Berlin.

Carnap, R., 1923. Über die Aufgabe der Physik und die Anwendung des Grundsatzes der Einfachstheit. Kant-Studien 28, 90-107.

Carnap, R., 1925. Über die Abhängigkeit der Eigenschaften des Raumes von denen der Zeit. Kant-Studien 30, 331-345.

Carnap, R., 1966. Philosophical Foundations of Physics: An Introduction to the Philosophy of Science. Basic Books, inc., New York.

Christoffel, E. B., 1869. Ueber die Transformation der homogenen Differentialausdrücke zweiten Grades. Journal für die reine und angewandte Mathematik 70, 46-70, now in Christoffel, 1910, vol I, 352-377, 378-382.

Corry, L., 2003. David Hilbert and the Axiomatization of Physics (1898-1918): from Grundlagen der Geometrie to Grundlagen der Physik. Kluwer, Dordrecht.

CPAE: Einstein, A., 1996-. The Collected Papers of Albert Einstein. Princeton University Press, Princeton.

de Sitter, W., 1916a. On Einstein's Theory of Gravitation, and its Astronomical Consequences (First paper). Monthly Notices of the Royal Astronomical Society 76 (9), 699-728.

de Sitter, W., 1916b. Space, Time, and Gravitation. The Observatory 39, 412-419.

Dieks, D., 2006. Another look at general covariance and the equivalence of reference frames. Studies in History and Philosophy of Modern Physics 37, 174-191.

DiSalle, R., 2006. Understanding Space-Time: The Philosophical Development of Physics from Newton to Einstein. Cambridge University Press, Cambridge, UK ; New York.

Earman, J., 1989. World Enough and Space-Time: Absolute vs. Relational Theories of Space and Time. MIT Press, Cambridge, Mass. 
Earman, J., 2002. Thoroughly Modern McTaggart: Or, What McTaggart Would Have Said if He Had Read the General Theory of Relativity. Philosophers' Imprint 2, 1-28.

Earman, J., Glymour, C., 1978. Einstein and Hilbert: Two Months in the History of General relativity. Archive for History of Exact Sciences 19, 291-308.

Earman, J., Norton, J. D., 1987. What Price Substantivalism. The Hole Story. British Journal for the Philosophy of Science 38, 515-525.

Eddington, A. S., 1923. The Mathematical Theory of Relativity. University Press, Cambridge.

Ehlers, J., Pirani, F. A. E., Schild, A., 1972. The Geometry of Free Fall and Light Propagation. In: O'Raifeartaigh, L. (Ed.), General Relativity. Papers in Honour of J. L. Synge. Clarendon Press, Oxford.

Einstein, A., 1913. Zum gegenwärtigen Stande des Gravitationsproblems. Physikalische Zeitschrift 14, 1249-1266, now in CPAE 4, Doc. 17.

Einstein, A., 1914a. Die formale Grundlage der allgemeinen Relativitätstheorie. Preussische Akademie der Wissenschaften, Sitzungsberichte -, 1030-1085, now in CPAE 6, Doc. 9.

Einstein, A., 1914b. Prinzipielles zur verallgemeinerten Relativitätstheorie. Physikalische Zeitschrift 15, 176-180, now in CPAE 4, Doc. 25.

Einstein, A., 1914c. Zum Relativitäts-Problem. Scientia (Bologna) 15, 337-348, now in CPAE 4, Doc. 31.

Einstein, A., 1915a. Erklärung der Perihelbewegung des Merkur aus der allgemeinen Relativitätstheorie. Preussische Akademie der Wissenschaften, Sitzungsberichte -, 831-839, now in CPAE 6, Doc. 24.

Einstein, A., 1915b. Feldgleichungen der Gravitation. Preussische Akademie der Wissenschaften, Sitzungsberichte -, 844-847, now in CPAE 6, Doc. 25.

Einstein, A., 1915c. Grundgedanken der allgemeinen Relativitätstheorie und Anwendung dieser Theorie in der Astronomie. Preussische Akademie der Wissenschaften, Sitzungsberichte -, 315 .

Einstein, A., 1915d. Zur allgemeinen Relativitätstheorie. Preussische Akademie der Wissenschaften, Sitzungsberichte -, 778-786, 799-801, now in CPAE 6, Doc. 21 and 22.

Einstein, A., 1916. Die Grundlage der allgemeinen Relativitätstheorie. Annalen der Physik 49, 769-822, now in CPAE 6, Doc 30.

Einstein, A., 1917. Über die spezielle und die allgemeine Relativitätstheorie (gemeinverständlich). Vieweg, Braunschweig, now in CPAE 6, Doc. 42.

Einstein, A., 1918. Prinzipielles zur allgemeinen Relativitätstheorie. Annalen der Physik 55, 241-244, now in CPAE 7, Doc. 4.

Einstein, A., 1952. Über die spezielle und die allgemeine Relativitätstheorie (gemeinverständlich), 16th Edition. Vieweg, Braunschweig.

Einstein, A., Grossmann, M., 1913. Entwurf einer verallgemeinerten Relativitätstheorie und eine Theorie der Gravitation. I. Physikalischer Teil von A. Einstein II. Mathematischer Teil von M. Grossmann. Teubner, Leipzig, now in CPAE 4, Doc. 13.

Einstein, A., Grossmann, M., 1914a. Entwurf einer verallgemeinerten Relativitätstheorie und eine Theorie der Gravitation. I. Physikalischer Teil von A. Einstein II. Mathematischer Teil von M. Grossmann. Zeitschrift für Mathematik und Physik 62, 225-244, 245-261, with additional "Comments" ("Bemerkungen") now in CPAE 4, Doc. 26. 
Einstein, A., Grossmann, M., 1914b. Kovarianzeigenschaften der Feldgleichungen der auf die verallgemeinerte Relativitätstheorie gegründeten Gravitationstheorie. Zeitschrift für Mathematik und Physik 63, 215-225, now in CPAE 6, Doc. 2.

Engler, F. O., 2006. Moritz Schlick und Albert Einstein, http://www.mpiwgberlin.mpg.de/Preprints/P309.PDF.

Frank, P., 1917. Die Bedeutung der physikalischen Erkenntnistheorie Machs für das Geistesleben der Gegenwart. Naturwissenschaften 5, 65-72, translated in Frank, 1950, pp. 6178 .

Frank, P., 1949. Einstein's Philosophy of Science. Reviews of Modern Physics 21 (3), 349-355.

Frank, P., 1950. Modern Science and its Philosophy. Harvard Univ. Press, Cambridge.

Friedman, M., 1983. Foundations of Space-Time Theories: Relativistic Physics and Philosophy of Science. Princeton University Press, Princeton.

Friedman, M., 1984. Roberto Torretti, Relativity and Geometry. Noûs 18 (14), 654-664.

Friedman, M., 1999. Reconsidering Logical Positivism. Cambridge University Press, New York.

Gabriel, G., 2004. Introduction: Carnap Brought Home. In: Awodey, S., Klein, C. (Eds.), Carnap Brought home: the View from Jena. Open Court, Chicago.

Giere, R. N., Richardson, A. W. (Eds.), 1996. Origins of Logical Empiricism. University of Minnesota Press, Minneapolis.

Habe, W. (Ed.), 1955. Wer ist wer? Vol. 12. Arani-Verlag, Berlin.

Hausdorff, F., 1914. Grundzüge der Mengenlehre. Von Veit, Leipzig.

Hawking, S. W., Ellis, G. F. R., 1973. The Large Scale Structure of Space-Time. Cambridge Univ. Press, Cambridge.

Hecht, H., Hoffmann, D., 1982. Die Berufung Hans Reichenbachs an die Berliner Universität. Deutsche Zeitschrift für Philosophie 30, 651-662.

Heidelberger, M., 2007. From Neo-Kantianism to Critical Realism: Space and the Mind-Body Problem in Riehl and Schlick. Perspectives on Science 15 (1), 26-48.

Helmholtz, H., 1870/1883. Über Ursprung und Bedeutung der Geometrischen Axiome. In: Vorträge und Reden. Vieweg, Braunschweig; Wiesbaden, pp. 1-31.

Helmholtz, H., 1921. Schriften zur Erkenntnistheorie. Springer, Berlin.

Helmholtz, H. v., 1977. Epistemological Writings. Reidel, Dordrecht, trans. by Malcolm F. Lowe; ed. by Robert S. Cohen and Yehuda Elkana.

Hentschel, K., 1986. Die Korrespondenz Einstein-Schlick: Zum Verhältnis der Physik zur Philosophie. Annals of Science 43, 475-488.

Hilbert, D., 1902. Über die Grundlagen der Geometrie. Göttiger Nachrichten -, 233-241.

Hilbert, D., 1903. Die Grundlagen der Geometrie, 2nd Edition. Teubner, Leipzig.

Hilbert, D., 1915. Grundlagen der Physik, Erste Mitteilung, vorgelegt in der Sitzung vom 20. November 1915. Nachrichten von der Königl. Gesellschaft der Wissenschaften und der Universität zu Göttingenn Math-physik. Klasse, 1915 -, 395-407, now in HIlbert 2009, ch. I, 28-46. 
Hilbert, D., Wintersemester 1916/1917. Die Grundlagen der Physik II, georg-AugustUniversität Göttingen, Mathematisches Institut, Lesesaal (now in Hilbert 2009, 162-307).

Hilbert, D., 1917. Grundlagen der Physik, Zweite Mitteilung, vorgelegt in der Sitzung vom 23. Dezember 1916. Nachrichten von der Königl. Gesellschaft der Wissenschaften und der Universität zu Göttingenn Math-physik. Klasse, 1915 -, 53-72, now in Hilbert 2009, ch. I, 47-72.

Hilbert, D., 2009. David Hilbert's Lectures on the Foundations of Physics 1915-1927. Springer Berlin Heidelberg.

Hoffmann, B., 1982. Some Einstein Anomalies. In: Holton, G., Elkana, Y. (Eds.), Albert Einstein: Historical and Cultural Perspectives. Dover Science Books. Dover Publications, pp. $91-105$.

Howard, D., 1984. Realism and Conventionalism in Einstein's Philosophy of Science: The Einstein-Schlick Correspondence. Philosophia naturalis 21, 618-29.

Howard, D., 1991. Einstein and Eindeutigkeit: A Neglected Theme in the Philosophical Background to General Relativity. In: Eisenstaedt, J., Kox, A. J. (Eds.), Historical Studies in General Relativity. Birkhäuser, Boston.

Howard, D., 1994. Einstein, Kant and the Origins of Logical Empiricism. In: Salmon, W. C., Wolters, G. (Eds.), Logic, Language, and the Structure of Scientific theories: Proceedings of the Carnap-Reichenbach Centennial, University of Konstanz, 21-24 May 1991. University of Pittsburgh Press; Universitätsverlag Konstanz, Pittsburgh, Pa.; Konstanz.

Howard, D., 1996. Relativity, Eindeutigkeit, and Monomorphism: Rudolf Carnap and the Development of the Categoricity Concept in Formal Semantics. In: Giere, R. N., Richardson, A. W. (Eds.), Origins of Logical Empiricism. University of Minnesota Press, Minneapolis.

Howard, D., 1999. Point Coincidences and Pointer Coincidences. Einstein on the Invariant Content of Space-Time Theories. In: Goenner, H. (Ed.), The Expanding Worlds of General Relativity. Birkhäuser, Basel, pp. 463-500.

Howard, D., Norton, J. D., 1993. Out of the Labyrinth. Einstein, Hertz, and the Gottingen Answer to the Hole Argument. In: The Attraction of Fravitation: New Studies in the History of General Relativity. Birkhäuser, Basel, pp. 30-62.

Huijnen, P., Kox, A., 2007. Paul Ehrenfest's Rough Road to Leiden: A Physicist's Search for a Position, 1904-1912. Physics in Perspective (PIP) 9, 186-211.

Illy, J., 1989. Einstein Teaches Lorentz, Lorentz Teaches Einstein their Collaboration in General Relativity, 1913-1920. Archive for History of Exact Sciences 39, 247-289.

Janssen, M. ., 1992. H. A. Lorentz's Attempt to Give a Coordinate-free Formulation of the General Theory of Relativity. In: Eisenstaedt, J., Kox, A. (Eds.), Studies in the History of General Relativity. Birkhäuser., Boston, pp. 344-363.

Janssen, M., 2003. A Glimpse Behind the Curtain of the Wizard/Un coup d'œil derrière le rideau du magicien. In: Lhéritier, G. (Ed.), The Einstein-Besso Manuscript: From Special Relativity to General Relativity/Le manuscrit Einstein-Besso: de la relativité restreinte à la relativité générale. Scriptura and Aristophile, Paris.

Janssen, M., 2007. What did Einstein know and when did He know it? A Besso Memo Dated August 1913. In: Janssen, M., Norton, J. D., Renn, J., Sauer, T., Stachel, J. (Eds.), The Genesis of General Relativity. Vol. 250 of Boston Studies in the Philosophy and History of Science. Springer Netherlands, pp. 787-837. 
Janssen, M., Renn, J., 2007. Untying the Knot: how Einstein Found his way Back to Field Equations Discarded in the Zurich Notebook. In: Janssen, M., Norton, J. D., Renn, J., Sauer, T., Stachel, J. (Eds.), The Genesis of General Relativity. Vol. 250 of Boston Studies in the Philosophy of Science. Springer Netherlands, pp. 839-925.

Komar, A., 1958. Construction of a Complete Set of Independent Observables in the General Theory of Relativity. Phys. Rev. 111, 1182-1187.

Kox, A., 1987. Hendrik Antoon Lorentz, the Ether, and the General Theory of Relativity. Archive for History of Exact Sciences 38 (1), 67-78.

Kretschmann, E., 1914. Eine Theorie der Schwerkraft in Rahmen der Ursprünglichen Einsteinen Relativitätstheorie. Ph.D. thesis, Friederich-Wilehelms-Univeristät zu Berlin, Berlin.

Kretschmann, E., 1915. Über die prinzipielle Bestimmbarkeit der berechtigten Bezugssysteme beliebiger Relativitätstheorien (I and II). Annalen der Physik 23, 907-942; 943-982.

Kretschmann, E., 1918. Über den physikalischen Sinn der Relativitätspostulate. A. Einsteins neue und seine ursprüngliche Relativitätstheorie. Annalen der Physik 53, 575-614.

Kretschmann, E., 1920. Der Liouvillesche Satz und die Relativitäatstheorie. Physik. Zs. 21, $484-487$.

Kretschmann, E., 1924. Das Maxwell-Boltzmannsche Geschwindigkeits- und Energieverteilungsgesetz in der Relativitätstheorie. Phys. Zs. 25, 162-165.

Kretschmann, E., 1930. Atom und Welle. Schriften der Königsberger Gelehrten Gesellschaft 6, 216-231, vortrag, gehalten am 7. Juli 1929 in öffentlicher Sitzung der Königsberger Gelehrten Gesellschaft.

Levi-Civita, T., Ricci-Curbastro, G., 1900. Méthodes de calcul différentiel absolu et leurs applications. Mathematische Annalen 54, 125-201, now in Ricci-Curbarstro 1956-57, I,.

Lorentz, H. A., 1917. On Einstein's Theory of Gravitation I. Proc. Royal Acad. Amsterdam 19 (II), 1341-1354.

Lorentz, H. A., 1923. The Determination of the Potentials in the General Theory of Relativity, with Some Remarks about the Measurement of Lengths and Intervals of Time and about the Theories of Weyl and Eddington. Proc. Royal Acad. Amsterdam 29, 383-399.

Mach, E., 1905. Erkenntnis und Irrtum. Skizzen zur Psychologie der Forschung. Barth, Leibzig.

Mach, E., 1906. Space and Geometry in the Light of Physiological, Psychological and Physical Inquiry. The Open Court Pub. Co., Chicago.

Maltese, G., 1991. The Rejection of the Ricci Tensor in Einstein's First Tensorial Theory of Gravitation. Archive for History of Exact Sciences 41 (4), 363-381.

Mancosu, P., Ryckman, T., 2002. Mathematics and Phenomenology: The Correspondence Between O. Becker and H. Weyl. Philosophia Mathematica 10 (2), 130-202.

Mancosu, P., Ryckman, T., 2005. Geometry, Physics and Phenomenology: the Correspondence between O. Becker and H. Weyl. In: Peckhaus, V. H. (Ed.), Oskar Becker und die Philosophie der Mathematik. Neuzeit \& Gegenwart. Fink, München.

Mehra, J., 1974. Einstein, Hilbert, and the Theory of Gravitation : Historical Origins of General Relativity Theory. Reidel, Dordrecht [et al.] 
Meyenn, K., 2009. Wolfgang Pauli's Philosophical Ideas Viewed from the Perspective of His Correspondence. In: Atmanspacher, H., Primas, H. (Eds.), Recasting Reality: Wolfgang Pauli's Philosophical Ideas and Contemporary Science. Springer Berlin Heidelberg, pp. $11-32$.

Minkowski, H., 1909. Raum und Zeit. Jahresberichte der Deutschen Mathematiker- Vereinigung, Leipzig, 1909. Teubner, Leibzig.

Moore, G. H., 2007. The Evolution of the Concept of Homeomorphism. Historia Mathematica $34,333-343$

Mormann, T., 2009. Geometrical Leitmotifs in Carnap's Early Philosophy. In: Friedman, M. (Ed.), The Cambridge Companion to Carnap. Cambridge University Press, Cambridge, pp. 43-64.

Norton, J., 1985. What was Einstein's Principle of Equivalence? Studies in History and Philosophy of Science Part A 16 (3), 203-246.

Norton, J., 2005. Conjecture on Einstein, the Independent Reality of Spacetime Coordinate Systems and the Disaster of 1913. In: Kox, A. J., Einsenstaedt, J. (Eds.), The Universe of General Relativity. Einstein Studies Volume 11. Birkhäuser, Boston, pp. 67-102.

Norton, J. D., 1984. How Einstein found his Field Equations: 1912-1915. Historical Studies in the Physical Sciences 14, 253-316.

Norton, J. D., 1995. Did Einstein Stumble? The Debate over General Covariance. Erkenntnis $42(2), 223-245$.

Norton, J. D., 1999. Geometries in Collision: Einstein, Klein and Riemann. In: Gray, J. (Ed.), The Symbolic Universe. Oxford University Press, Oxford; New York, pp. 128-144.

Norton, J. D., 2003. General Covariance, Gauge Theories, and the Kretschmann Objection. In: Brading, K., Castellani, E. (Eds.), Symmetries in Physics. Philosophical Reflections. Cambridge University Press, Cambridge, pp. 110-123.

Norton, J. D., 2007. What was Einstein's "Fateful Prejudice"? In: Janssen, M., Norton, J. D., Renn, J., Sauer, T., Stachel, J. (Eds.), The Genesis of General Relativity. Vol. 250 of Boston Studies in the Philosophy of Science. Springer Netherlands, pp. 715-783.

Pais, A., 1982. Subtle is the Lord: The Science and the Life of Albert Einstein. Oxford University Press, New York.

Parrini, P., 2005. L'empirismo logico. Aspetti storici e prospettive teoriche. Carocci, Roma.

Pauli, W., 1921. Relativitätstheorie. In: Enzyklopädie der mathematischen Wissenschaften. Vol. V/19. Teubner, pp. 539-775, repnnted in book form (Leipzig: Teubner, 1921).

Petzoldt, J., 1921. Die Stellung der Relativitätstheorie in der geistigen Entwicklung der Menschheit, 1st Edition. Sibyllen-Verlag, Dresden.

Pitts, J. B., 2008. General Covariance, Artificial Gauge Freedom And Empirical Equivalence. Ph.D. thesis, University of Notre Dame.

Poincaré, H. ., 1906a. Wissenschaft und Hypothese. Teubner, Leipzig.

Poincaré, H., 1895. Analysis Situs. Journal de l'École Polytechnique 2 (1), 1-123.

Poincaré, H., 1902. La science et l'hypothèse. Flammarion, Paris.

Poincaré, H., 1905. La valeur de la science. Flammarion, Paris. 
Poincaré, H., 1906b. Der Wert der Wissenschaft. B.G. Teubner, Leipzig.

Poincaré, H., 1906c. Sur la dynamique de l'électron. Rendiconti del circolo matematico di Palermo 21, 129-176.

Poincaré, H., 1913a. Dernières pensées. E. Flammarion, Paris.

Poincaré, H., 1913b. Letzte Gedanken. Akademische Verlagsgesellschaft, Leipzig.

Poincaré, H., 1963. Mathematics and Science: Last Essays. Dernières pensées. Dover Publications, New York,.

Popper, K., 1929. Axiome, Definitionen und Postulate der Geometrie, in The Karl Popper Archives (4.6), now in Popper, 2006.

Popper, K. R., 2006. Frühe Schriften. Mohr Siebeck, Tübingen.

Reich, K., 1994. Die Entwicklung des Tensorkalküls: vom absoluten Differentialkalkül zur Relativitätstheorie. Birkhäuser, Berlin.

Reichenbach, H., 1920. Relativitätstheorie und Erkenntnis apriori. Springer.

Reichenbach, H., 1920/1965. The Theory of Relativity and a priori Knowledge. University of California Press, Berkeley.

Reichenbach, H., 1922a. Der gegenwärtige Stand der Relativitätsdiskussion. Eine kritische Untersuchung. Logos 22 (10), 316-378, now in Reichenbach, 1977, III.

Reichenbach, H., 1922b. La signification philosophique de la théorie de la relativité. Revue philosophique de la France et de l'Étranger 93, 5-61.

Reichenbach, H., 1924. Axiomatik der relativistischen Raum-Zeit-Lehre. Braunschweig, Vieweg.

Reichenbach, H., 1928. Philosophie der Raum-Zeit-Lehre. Walter de Gruyter, Berlin and Leipzig, now in Reichenbach 1977, vol II.

Reichenbach, H., 1944. Philosophical Foundations Of Quantum Mechanics. University of California Press, Berkely-Los Angeles-London.

Reichenbach, H., 1949. The Philosophical Significance of the Theory of Relativity. In: Schilpp, P. A. (Ed.), Albert Einstein. Philosopher-Scientist. Tudor, New York, pp. 289-311.

Reichenbach, H., 1951. The Rise of Scientific Philosophy. University of California Press, Berkely/Los Angeles/London.

Reichenbach, H., 1958. The Philosophy of Space and Time. Dover Publ., New York.

Reichenbach, H., 1965. The Theory of Relativity and a priori Knowledge. University of California Press, Berkeley.

Reichenbach, H., 1969. Axiomatization of the Theory of Relativity. University of California Press, Berkeley,.

Reichenbach, H., 1977. Gesammelte Werke in 9 Bänden. Vieweg, Braunschweig; Wiesbaden.

Reichenbach, H., 1978. Selected Writings: 1909 - 1953. Vienna circle collection ; 4. Reidel, Dordrecht.

Renn, J., Sauer, T., 2007. Pathways out of Classical Physics: Einstein's Double Strategy in his Search for the Gravitational Field Equation. In: Janssen, M., Norton, J., Renn, J., Sauer, T., Stachel, J. (Eds.), The Genesis of General Relativity. Vol. 250 of Boston Studies in the Philosophy of Science. Springer Netherlands, pp. 113-312. 
Renn, J., Stachel, J., 2007. Hilbert's Foundation of Physics: From a Theory of Everything to a Constituent of General Relativity. In: Renn, J., Janssen, M. (Eds.), The Genesis of General Relativity. Springer Netherlands, pp. 857-972.

Renn, J. e. a., 2007. The Genesis of General relativity. Springer, Dordrecht.

Rickles, D., 2008. Symmetry, Structure and Spacetime. Elsevier, Amsterdam.

Riehl, A., 1876-1887. Der philosophische Kriticismus und seine Bedeutung für die positive Wissenschaft. Vol. 1, Geschichte und Methode des philosophischen Kriticismus (1876); vol. 2, part 1 Die sinnlichen und logischen Grundlagen der Erkenntnis (1879); vol. 2, part 2 Zur Wissensckaßstheorie und Metaphysik (1887). Wilhelm Engelmann, Leipzig.

Riehl, A., 1908-1926. Der philosophische Kritizismus. Geschichte und System, 2nd Edition. Vol. 1 (1908), Geschichte des philosophischen Kritizismus. Vol. 2, Die sinnlichen und logischen Grundlagen der Erkenntnis, ed. Hans Heyse and Eduard Spranger (Leipzig: Kröner, 1925). Vol. 3, Zur Wissenschaftstheorie und Metaphysik, ed., Hans Heyse and Eduard Spranger (Leipzig: Kröner, 1926). Engelmann, Leipzig.

Rovelli, C., 2004. Quantum gravity. Cambridge University Press, Cambridge, UK ; New York.

Ryckman, T., 1992. (P)oint-(C)oincidence Thinking. The Ironical Attachment of Logical Empiricism to General Relativity. Studies in History and Philosophy of Modern Physics 23 (3), 471-497.

Ryckman, T., 2005. The Reign of Relativity. Philosophy in Physics 1915-1925. Oxford University Press, Oxford, New York.

Ryckman, T., 2007. Logical Empiricism and Philosophy of Physics. In: Richardson, A. W., Uebel, T. (Eds.), The Cambridge Companion to Logical Empiricism, 1st Edition. Cambridge University Press, Cambridge [et al.], pp. XI, 430 S.

Rynasiewicz, R., 1999. Kretschmann's Analysis of Covariance and Relativity Principles. In: Goenner, H. (Ed.), The Expanding Worlds of General Relativity. Birkhäuser, Basel, pp. 431-462.

Sauer, T., 1999. The Relativity of Discovery: Hilbert's First Note on the Foundations of Physics. Archive for History of Exact Sciences 53, 529-575.

Schilpp, P. A. (Ed.), 1949. Albert Einstein: Philosopher Scientist. Library of Living Philosophers, Evanston.

Schlick, M., 1904. Über die Reflexion des Lichtes in einer inhomogenen Schicht. Ph.D. thesis, Friederich-Wilehelms-Univeristät zu Berlin, Berlin.

Schlick, M., 1915. Die philosophische Bedeutung des Relativitätsprinzips. Zeitschrift für Philosophie und philosophische Kritik 159, 129-175.

Schlick, M., 1917a. Raum und Zeit in der gegenwärtigen Physik. Zur Einführung in das Verständnis der allgemeinen Relativitätstheorie. Die Naturwissenschaften 5, 161-167; 177-186, now in Schlick, 2006, vol. II.

Schlick, M., 1917b. Raum und Zeit in der gegenwärtigen Physik. Zur Einführung in das Verständnis der allgemeinen Relativitätstheorie. Springer, Berlin, now in Schlick, 2006, vol. II.

Schlick, M., 1918. Allgemeine Erkenntnisslehre. Naturwissenschaftliche Monographien und Lehrbücher. J. Springer, Berlin, now in Schlick 2006-, vol. I. 
Schlick, M., 1919. Raum und Zeit in der gegenwärtigen Physik : zur Einführung in das Verständnis der Relativitäts- und Gravitationstheorie, 3rd Edition. Springer, Berlin, now in Schlick, 2006, vol. II.

Schlick, M., 1920. Raum und Zeit in der gegenwärtigen Physik. Zur Einführung in das Verständnis der allgemeinen Relativitätstheorie, 3rd Edition. Springer, Berlin, now in Schlick, 2006, vol. II.

Schlick, M., 1925. Allgemeine Erkenntnisslehre, 2nd Edition. Naturwissenschaftliche Monographien und Lehrbücher. J. Springer, Berlin, now in Schlick 2006, vol. I.

Schlick, M., 1978. Philosophical Papers. Vienna Circle Collection, 11. Reidel, Dordrecht.

Schlick, M., 2006-. Gesamtausgabe. Springer, Berlin.

Schlick, M., Reichenbach, H., 1920-22. Correspondence 1920-22, http://echo.mpiwgberlin.mpg.de/content/space/space/reichenbach1920-22.

Stachel, J., 1980. Einstein's Search for General Covariance, 1912-1915, the paper was first read at the Ninth International Conference on General Relativity and Gravitation, Jena in 1980; reprented in Stachel, 2002, 301-337.

Stachel, J., 1980/2002. Einstein's Search for General Covariance, 1912-1915. In: Einstein from "B" to "Z". Birkhäuser, Boston, pp. 301-337, the paper was first read at the Ninth International Conference on General Relativity and Gravitation, Jena in 1980.

Stachel, J., 1987. How Einstein Discovered General Relativity: a Historical Tale with Some Contemporary Morals. In: MacCallum, M. (Ed.), General Relativity and Gravitation. Proceedings of the 11th International Conference on General Relativity and Gravitations. Cambridge University Press, Cambridge, pp. 200-208, republished in Stachel, 2002, 293-300.

Stachel, J., 1988/1992. The Cauchy Problem in General Relativity-The Early Years. In: Eisenstaedt, J. H. (Ed.), Studies in the History of General Relativity: Based on the Proceedings of the 2nd International Conference on the History of General Relativity, Luminy, France, 1988. Birkhäuser, Boston [et al.], pp. 407-418.

Stachel, J., 1993. The Meaning of General Covariance: The Hole Story. In: Earman, J. (Ed.), Philosophical Problems of the Internal and External Worlds : Essays on the Philosophy of Adolf Grünbaum. Pittsburgh-Konstanz series in the philosophy and history of science. Univ. of Pittsburgh Pr., Pittsburgh, Pa., pp. 129-160, festschrift Adolf Grünbaum.

Stachel, J. J., 2002. Einstein from "B" to "Z". Einstein studies ; 9. Birkhäuser, Boston.

Study, E., 1914. Die realistische Weltansicht und die Lehre von Raume: Geometrie, Anschauung und Erfahrung. F. Vieweg \& Sohn, Braunschweig.

Weyl, H., 1913. Die Idee der Riemannschen Fläche. Teubner, Leipzig und Berlin, based on Weyl's Lectures at the University of Göttingen (Winter Term 1911-12).

Weyl, H., 1918. Raum, Zeit, Materie: Vorlesungen über allgemeine Relativitätstheorie. Springer, Berlin.

Weyl, H., 1920. Die Diskussion über die Relativitätstheorie. Die Umschau 24, 609-611.

Weyl, H., 1921. Zur Infinitesimalgeometrie: Einordnung der projektiven und konformen Auffassung. Nachrichten der Königlichen Gesellschaft der Wissenschaften zu Göttingen. Mathematisch Physikalische Klasse, 99-112Now in WGA II, Doc. 43.

Weyl, H., 1924. Massenträgheit und Kosmos. Ein Dialog. Naturwissenschaften 12, 197-204, now in WGA II, Doc. 65.

Weyl, H., 1931. Geometrie und Physik. Die Naturwissenschaften 19, 49-58.

WGA: Weyl, H., 1968. Gesammelte Abhandlungen. Springer, Berlin. 